\title{
Eclipses by circumstellar material in the T Tauri star AA Tau
}

\section{Evidence for non-stationary magnetospheric accretion ${ }^{\star}$}

\author{
J. Bouvier ${ }^{1}$, K. N. Grankin ${ }^{2}$, S. H. P. Alencar ${ }^{3}$, C. Dougados ${ }^{1}$, M. Fernández ${ }^{4}$, G. Basri ${ }^{5}$, C. Batalha ${ }^{6}$, E. Guenther ${ }^{7}$, \\ M. A. Ibrahimov ${ }^{2}$, T. Y. Magakian ${ }^{8}$, S. Y. Melnikov ${ }^{2}$, P. P. Petrov ${ }^{9}$, M. V. Rud ${ }^{10}$, and M. R. Zapatero Osorio ${ }^{11}$ \\ 1 Laboratoire d'Astrophysique, Observatoire de Grenoble, Université Joseph Fourier, BP 53, 38041 Grenoble Cedex 9, France \\ 2 Astronomical Institute of the Academy of Sciences of Uzbekistan, Astronomicheskaya 33, Tashkent 700052, Uzbekistan \\ 3 Departamento de Física - ICEx - UFMG, Caixa Postal 702, 30161-970, Belo Horizonte, Brasil \\ 4 Instituto de Astrofísica de Andalucía, CSIC, Apdo. 3004, 18080 Granada, Spain \\ 5 Department of Astronomy, University of California at Berkeley, 601 Campbell Hall 3411, Berkeley, CA 94720, USA \\ ${ }^{6}$ Observatório Nacional/CNPq, Rua General José Cristino 77, Rio de Janeiro, RJ 20920-400, Brazil \\ 7 Thüringer Landessternwarte Tautenburg, Karl-Schwarzschild-Observatorium, Sternwarte 5, 07778 Tautenburg, Germany \\ 8 Byurakan Astrophysical Observatory, Aragatsotn prov., 378433 Armenia \\ 9 Crimean Astrophysical Observatory and Isaac Newton Institute of Chile, Crimean Branch, p/o Nauchny, Crimea 98409, \\ Ukraine \\ 10 Fessenkov Astrophysical Institute, 480068 Almaty, Kazakstan \\ 11 LAEFF-INTA, PO Box 50727, 28080 Madrid, Spain
}

Received 14 May 2003 / Accepted 18 June 2003

\begin{abstract}
We report the results of a synoptic study of the photometric and spectroscopic variability of the classical T Tauri star AA Tau on timescales ranging from a few hours to several weeks. The AA Tau light curve had been previously shown to vary with a $8.2 \mathrm{~d}$ period, exhibiting a roughly constant brightness level, interrupted by quasi-cyclic fading episodes, which we interpreted as recurrent eclipses of the central star by the warped inner edge of its accretion disk (Bouvier et al. 1999). Our observations show the system is dynamic and presents non-stationary variability both in the photometry and spectroscopy.

The star exhibits strong emission lines that show substantial variety and variability in their profile shapes and fluxes. Emission lines such as $\mathrm{H} \alpha$ and $\mathrm{H} \beta$ show both infall and outflow signatures and are well reproduced by magnetospheric accretion models with moderate mass accretion rates $\left(10^{-8}-10^{-9} M_{\odot} \mathrm{yr}^{-1}\right)$ and high inclinations $\left(i \geq 60^{\circ}\right)$. The veiling shows variations that indicate the presence of 2 rotationally modulated hot spots corresponding to the two magnetosphere poles. It correlates well with the He I line flux, with $B-V$ and the $V$ excess flux. We have indications of a time delay between the main emission lines ( $\mathrm{H} \alpha, \mathrm{H} \beta$ and $\mathrm{He} \mathrm{I}$ ) and veiling, the lines formed farther away preceding the veiling changes. The time delay we measure is consistent with accreted material propagating downwards the accretion columns at free fall velocity from a distance of about $8 R_{\star}$. In addition, we report periodic radial velocity variations of the photospheric spectrum which might point to the existence of a $0.02 M_{\odot}$ object orbiting the star at a distance of $0.08 \mathrm{AU}$.

During a few days, the eclipses disappeared, the variability of the system was strongly reduced and the line fluxes and veiling severely depressed. We argue that this episode of quiescence corresponds to the temporary disruption of the magnetic configuration at the disk inner edge. The smooth radial velocity variations of inflow and outflow diagnostics in the $\mathrm{H} \alpha$ profile yield further evidence for large scale variations of the magnetic configuration on a timescale of a month. These results may provide the first clear evidence for large scale instabilities developping in T Tauri magnetospheres as the magnetic field lines are twisted by differential rotation between the star and the inner disk. The interaction between the inner accretion disk and the stellar magnetosphere thus appears to be a highly dynamical and time dependent process.
\end{abstract}

Key words. accretion, accretion disks - stars: pre-main sequence - stars: magnetic fields - stars: individual: AA Tau

Send offprint requests to: J. Bouvier,

e-mail: jbouvier@obs.ujf-grenoble.fr

* Based on observations obtained at Observatoire de Haute Provence (CNRS, France), Mt Maidanak Obs. (Uzbekistan), Calar Alto Obs. (Spain), Teide Obs. (Spain), Byurakan Obs. (Armenia), Assy-Turgen Obs. (Kazakstan), ESO La Silla (Chile), Lick Obs.

\section{Introduction}

$\mathrm{T}$ Tauri stars are low-mass stars with an age of a few million years at most, still contracting down their Hayashi tracks towards the main sequence. They are classified in two groups,

(NOAO, USA), Tautenburg Obs. (Germany) and Roque de los Muchachos Obs. (Spain). 
the weak-line T Tauri stars (WTTS) which merely exhibit enhanced solar-type activity and the classical $\mathrm{T}$ Tauri stars (CTTS) which actively accrete material from a circumstellar disk (see, e.g., Ménard \& Bertout 1999). Understanding the accretion process in young solar type stars, as well as the associated mass loss phenomenon, is one of the major goals in the study of T Tauri stars. Indeed, accretion has a significant and long lasting impact on the evolution of low mass stars by providing both mass and angular momentum, and the evolution and fate of circumstellar accretion disks around young stars has become an increasingly important issue since the discovery of extrasolar planets and planetary systems with unexpected properties. Deriving the properties of young stellar systems, of their associated disks and outflows is therefore an important step towards the establishment of plausible scenarios for star and planet formation.

Early models assumed that the accretion disk of CTTS extended all the way down to the star. However, the recognition that young stars have strong surface magnetic fields of order of 1-3 kG (Johns-Krull et al. 1999, 2001; Guenther et al. 1999; Smith et al. 2003) raised the issue of the impact an extended stellar magnetosphere might have on the structure of the inner disk. Assuming that the main component of the stellar magnetosphere on the large scale is a dipole, Königl (1991) showed that, for typical mass accretion rates in the disk $\left(10^{-9}\right.$ to $10^{-7} M_{\odot} \mathrm{yr}^{-1}$; Basri \& Bertout 1989; Hartigan et al. 1995; Gullbring et al. 1998), the magnetic torque exerted by the field lines onto the gaseous disk was comparable to the viscous torque due to turbulence in the disk at a few stellar radii (see also Camenzind 1990). Hence, the inner disk is expected to be truncated by the magnetosphere at a distance of a few stellar radii above the stellar surface. From there, material is channelled onto the star along the magnetic field lines, thus giving rise to magnetospheric accretion columns filled with hot plasma. As the accreted material in the funnel flow eventually hits the stellar surface at free fall velocity, strong accretion shocks develop near the magnetic poles.

Observational support for these predictions of the magnetospheric accretion scenario in CTTS has been accumulating over the last decade (see Bouvier et al. 2003 for a recent review). Magnetospheric cavities with inner radii in the range 3-8 $R_{\star}$ are called for to account for the near-IR properties of CTTS systems (Bertout et al. 1988; Meyer et al. 1997). Inverse P Cygni profiles observed in the Balmer and Pashen line profiles of CTTS, with redshifted absorption components reaching velocities of several hundred $\mathrm{km} \mathrm{s}^{-1}$, point to hot gas free falling onto the star from a distance of a few stellar radii (Edwards et al. 1994; Folha \& Emerson 2001). Magnetospheric accretion models have indeed been successful in reproducing the main characteristics of the emission line profiles of some CTTS, which suggest that at least part of the line emission arises in accretion columns (Hartmann et al. 1994; Muzerolle et al. 2001). Finally, hot spots covering about $1 \%$ of the stellar surface are thought to be responsible for the rotational modulation of CTTS luminosity (Bouvier \& Bertout 1989; Vrba et al. 1993) and are identified with the accretion shocks expected to develop near the magnetic poles in the magnetospheric accretion scenario.
The observational evidence for magnetically channelled accretion in CTTS has led to the development of steady-state axisymmetric MHD models which describe the interaction between the inner disk and a stellar dipole. These models provide a framework to understand the physical connection between accretion and mass loss in CTTS, with the open magnetic field lines threading the disk carrying away part of the accretion flow while the remaining part is channelled onto the star (e.g. Shu et al. 1994; Ferreira 1997). Synoptic studies of a few CTTS systems have revealed correlated time variability of the inflow and outflow diagnostics, both being modulated on a rotation timescale. This has been interpreted as evidence for an inclined stellar magnetosphere disrupting the inner disk (Johns \& Basri 1995a; Petrov et al. 1996; Oliveira et al. 2000; Petrov et al. 2001).

Magnetically mediated accretion in CTTS is presumably more complex and possibly much more time variable than depicted by axisymmetric, steady-state MHD models. Expanding upon earlier models (Aly \& Kuijpers 1990; van Ballegooijen 1994; Lynden-Bell \& Boily 1994), recent numerical simulations of the disk/magnetosphere interaction suggest that the magnetic field lines that connect the star to the disk can be substantially deformed by differential rotation on short timescales. One class of models thus predict that differential rotation between the footpoints of the field lines, one being anchored into the star the other into the disk, leads to field line expansion, opening and reconnection which eventually restores the initial (dipolar) configuration (e.g. Goodson et al. 1997; Goodson \& Winglee 1999). This magnetospheric inflation process is thus expected to be cyclic on a timescale of a few rotation periods and to be accompanied by both episodic outflows during the opening of the magnetic structure and time dependent accretion onto the star (Hayashi et al. 1996; Romanova et al. 2002). Other models, however, suggest that under the action of differential rotation the field lines drift radially outwards in the disk leading to magnetic flux expulsion (Bardou \& Heyvaerts 1996). The response of the magnetic configuration to differential rotation mainly depends upon magnetic diffusivity in the disk, a free parameter of the models which is unfortunately poorly constrained by current observations.

Due mostly to the lack of intense monitoring of CTTS on proper timescales, the observational evidence for a time dependent interaction between the inner disk and the stellar magnetosphere is at present quite limited. Episodic high velocity outbursts, possibly connected with magnetospheric reconnection events predicted by recent numerical simulations, have been reported for a few systems based on the slowly varying velocity shift of blueshifted absorption components of emission line profiles on a timescale of hours to days (Alencar et al. 2001; Ardila et al. 2002). Possible evidence for magnetic field lines being twisted by differential rotation between the star and the disk has been reported for SU Aur by Oliveira et al. (2000). Another possible evidence for magnetic field lines being twisted by differential rotation thus leading to quasiperiodic reconnection processes has been reported for the embedded protostellar source YLW 15 based on the observations of quasi-periodic X-ray flaring (Montmerle et al. 2000). 
Table 1. Journal of observations.

\begin{tabular}{|c|c|c|c|c|c|c|c|}
\hline \multicolumn{4}{|c|}{ Photometry } & \multicolumn{4}{|c|}{ Spectroscopy } \\
\hline $\begin{array}{l}\text { Dates } \\
\text { (1999) }\end{array}$ & Site & Telescope & Filters & $\begin{array}{l}\text { Dates } \\
\text { (1999) }\end{array}$ & Site & Telescope & $\begin{array}{l}\text { Spectral range } \\
(\AA)\end{array}$ \\
\hline $09 / 08-16 / 12$ & Mt Maidanak & $0.5 \mathrm{~m}$ & $U B V R$ & $29 / 11-16 / 12$ & OHP & $1.93 \mathrm{~m}$ & $3890-6800$ \\
\hline $29 / 11-15 / 12$ & OHP & $1.20 \mathrm{~m}$ & $U B V R I$ & $25-28 / 11,20-26 / 12$ & ESO, La Silla & $1.52 \mathrm{~m}$ & $3530-9200$ \\
\hline $25 / 11-02 / 12$ & Calar Alto & $1.50 \mathrm{~m}$ & $B V R I$ & $26-27 / 11,11-17 / 12$ & Lick & CAT & $3850-10650$ \\
\hline 26/11-04/01 & Teide & $0.80 \mathrm{~m}$ & $B V R I$ & $28-29 / 11,18 / 12$ & Lick & $3 \mathrm{~m}$ & $3850-10650$ \\
\hline $30 / 11-08 / 12$ & Byurakan & $2.6 \mathrm{~m}$ & $V R I$ & $27-28 / 11,16-21 / 12$ & Tautenburg & $2 \mathrm{~m}$ & $4680-7100$ \\
\hline 08/09-30/09 & Almaty & $1 \mathrm{~m}$ & $B V R$ & $27-28 / 11$ & Canarias & NOT & $4840-9400$ \\
\hline
\end{tabular}

Since magnetically dominated accretion occurs on a scale of a few stellar radii $(\leq 0.1 \mathrm{AU})$ which, at the distance of the nearest star forming region cannot be resolved yet by current telescopes, one of the most fruitful approach to probe the structure and evolution of this compact region is to monitor the variations of the system over several rotation timescales. We therefore started synoptic campaigns on a number of CTTS a few years ago. Results of previous campaigns have been reported by Chelli et al. (1999) for DF Tau and by Bouvier et al. (1999, hereafter B99) for AA Tau. The latter object proved to be ideally suited to probe the inner few 0.01 AU of the system: due to its high inclination $\left(i \simeq 75^{\circ}\right.$, see B99), the line of sight to the star intersects the region where the inner disk interacts with the stellar magnetosphere. The peculiar orientation of this otherwise typical CTTS maximizes the variability induced by the modulation of the magnetospheric structure and thus provides the strongest constraints on the inner disk and the magnetospheric cavity. During the first campaign (B99), multicolor photometry was obtained with no simultaneous spectroscopy. This led to the discovery of recurrent eclipses of the central object with a period of 8.2 days. We attributed these eclipses to the Keplerian rotation of a non axisymmetric warp at the inner disk edge which periodically obscures the line of sight to the star. We further proposed that the warped inner disk edge directly resulted from the interaction of the disk with an inclined magnetosphere, an expectation promptly confirmed by Terquem \& Papaloizou (2000, see also Lai 1999). While this first campaign provided insight into the structure of the inner disk on a scale of about $0.1 \mathrm{AU}$ and constrained the large scale structure of AA Tau's magnetosphere, the lack of simultaneous spectroscopy prevented us from investigating the accretion columns connecting the inner disk to the star.

We therefore organized a new campaign on AA Tau during the fall of 1999 combining simultaneous photometric and spectroscopic monitoring over several rotation periods. One goal was to further investigate the magnetospheric accretion region and relate the inner disk warp to accretion columns and accretion shocks in a consistent way. Another goal was to investigate the stability of the magnetospheric accretion process on a month timescale, the duration of the campaign, as well as on much longer timescales by comparing the results of the 2 campaigns separated by 4 years.

The results of the campaign performed in 1999 are described in this paper. Section 2 briefly describes the aquisition of photometric and spectroscopic observations at various observatories, over a period of 5 months for photometry and simultaneously over a period of 1 month for spectroscopy. Section 3 presents the results of the spectroscopic and photometric variability observed on timescales ranging from hours to months. Section 4 discuss the origin of the variability of the system and its relevance to the magnetospheric accretion process. We argue that the main source of photometric variability is variable circumstellar extinction which is ascribed, as for the previous campaign, to the recurrent occultation of the central star by the warped inner disk. The spectroscopic variability provides evidence for magnetospheric accretion columns and associated hot spots. In addition, we find that the accretion process is time dependent and smoothly varies on a time scale of a month. We argue that the time dependent accretion rate onto the star results from the development of large scale instabilities in the magnetospheric structure, reminiscent of the magnetospheric inflation cycles predicted by recent numerical simulations. Section 5 concludes that the interaction between the inner disk and the star's magnetosphere is a highy dynamical and time dependent process and mentions a few implications of this result.

\section{Observations}

We describe in this section the multi-site campaign of observations. Due to the numerous observatories involved and to the variety of intrumentation we used, we provide only a brief account of the data aquisition and reduction procedures at each site. The journal of the observations is given in Table 1 .

\subsection{Photometry}

The photometric observations were carried out over a period extending from Aug. 9, 1999, to Jan. 4, 2000. AA Tau's light curve is best sampled in the BVRI filters over the period from Nov. 25 to Dec. 15, 1999, when several sites observed simultaneously (see Table 1).

CCD photometry was performed at all sites except for Mt Maidanak and Assy-Turgen observatories where a photomultiplier tube was used. After images were suitably reduced (bias and flat-field corrected), differential photometry between AA Tau and two reference stars was obtained using the IRAF/DAOPHOT PSF fitting package. The two reference stars are located less than $2^{\prime}$ away from AA Tau and recorded 
on the same images (these are stars No. 1125-01689518 and $1125-01691043$ in the USNO2 catalogue). At Mt Maidanak observatory, absolute photometry of AA Tau was obtained in the $U B V R$ filters, thus providing the required calibration of the differential light curves derived from CCD photometry at other sites. The photometric zero points of the differential $U B V R$ light curves were thus derived from measurements obtained simultaneously at Mt Maidanak and OHP. Since no observations were performed in the $I$ band at Mt Maidanak, the differential $I$-band light curve cannot be calibrated this way. Instead, we assumed that the average $(V-I)$ color of AA Tau had not changed between 1995 (Bouvier et al. 1999) and these new observations. This assumption is supported by the fact that the average $(V-R)$ color of AA Tau has remained the same between the two epochs (see Sect. 3.1). The resulting calibrated light curves have an rms photometric error of order of $0.02 \mathrm{mag}$ in the $B V R_{\mathrm{c}} I_{\mathrm{c}}$ filters and up to $0.2 \mathrm{mag}$ in the $U$-filter due to the system's faintness at this wavelength. The I-band light curve might have a systematic photometric error up to $0.05 \mathrm{mag}$ due to the calibration method. The data are available electronically at CDS Strasbourg via anonymous ftp to cdsarc.u-strasbg.fr (130.79.128.5) or via http://cdsweb.u-strasbg.fr/cgi-bin/qcat?]/A+A/409/169.

Diaphragm photometry was performed at Maidanak Observatory (Uzbekistan) from Aug. 9 to Dec. 16, 1999, using the $48 \mathrm{~cm}$ telescope equipped with a FEU-79 tube. Measurements were obtained in the $U B V R$ bands with a diaphragm of $28^{\prime \prime}$. Integration times ranged from 50 up to $120 \mathrm{~s}$, depending on the filter. During one of the photometric nights of the run, secondary standards were observed. The data were reduced with standard procedures and assuming average extinction coefficients for the site. The final photometric error is about $0.01 \mathrm{mag}$.

UBVRI observations were collected from Nov. 29 to Dec. 15, 1999, at Observatoire de Haute-Provence (France) on the $1.2 \mathrm{~m}$ telescope. The detector was a $1 \mathrm{k}$ CCD camera yielding a field of view of $12^{\prime}$. The exposure time ranged from 20 to $120 \mathrm{~s}$ depending on the filter and adapted to seeing conditions in order to obtain a high signal to noise ratio on AA Tau and the two comparison stars. CCD images were biased corrected and flat-fielded with proper calibration images following a standard reduction procedure.

Observations in the BVRI Johnson-Cousins system were carried out from Nov. 25 to Dec. 2, 1999 at the $1.5 \mathrm{~m}$ telescope at the EOCA (Estación de Observación de Calar Alto, Almería, Spain) using a Tektronics TK1024AB CCD, $1024 \times 1024$ pixels with a field of view $6.9 \times 6.9^{\prime 2}$. Integration times for the $V R I$ filters were computed to maximize the $S / N$ ratio while remaining well within the linear region of the CCD.

Additional BVRI broad-band photometry of AA Tau was collected with the $0.8 \mathrm{~m}$ IAC80 telescope at Teide Observatory (Spain) on thirteen nights of November through December 1999 and January 2000. We used the Thomson detector $(1024 \times 1024$ pixel $)$ mounted at the Cassegrain focus of the telescope, which provides a pixel size of $0.43^{\prime \prime}$ and a field of view of $7.4 \times 7.4^{\prime 2}$. Exposure times were typically $120-200 \mathrm{~s}$ in the $B V$ bands, and 60-100 s in the $R I$ filters. Data were taken with a seeing of $1.5-2.0^{\prime \prime}$. We processed raw frames with usual techniques within the IRAF environment, which included bias subtraction, flat-fielding and correction for bad pixels by interpolation with values from the nearest-neighbour pixels.

$B V R$ observations of AA Tau were conducted with the $1.0 \mathrm{~m}$ "Carl Zeiss" Jena telescope at the Assy-Turgen Observatory (2600 m altitude) near Almaty (Kazakstan) during four nights in September 1999. The single channel photometerpolarimeter with the photocathode S20 operating in photon counting mode was attached to the Cassegrain focus of the telescope. Exposure times were 60s through a diaphragm of $20^{\prime \prime}$.

Photometric observations in Byurakan (Armenia) were performed on 3 nights between Nov. 30 and Dec. 8, 1999. The $2.6 \mathrm{~m}$ telescope was equipped with the ByuFOSC-2 spectral camera, equipped with a $1060 \times 514$ CCD (Movsessian et al. 2000) working in the imaging mode with a $12^{\prime} \times 6^{\prime}$ field of view. Images were obtained in VRI filters and were bias subtracted and flat-field calibrated following a standard procedure.

\subsection{Spectroscopy}

The spectroscopic observations were carried out from Nov. 25 to Dec. 26, 1999, from 5 sites. We obtained 54 highresolution echelle spectra of AA Tau over this period in quasisimultaneity with the photometry (see Table 1). Integration times ranged between 3600 and 5400 s yielding an average $S / N \simeq 30$ on the red continuum.

We obtained 23 spectra at the $1.93 \mathrm{~m}$ OHP telescope with the ELODIE dual fiber echelle spectrograph (Baranne et al. 1996) which yields 67 orders covering the $3906 \AA$ to $6811 \AA$ domain at a mean spectral resolution of $\lambda / \Delta \lambda \simeq 42000$, and records simultaneously the object and the neighboring sky. Spectra were reduced with the automatic on-line TACOS software (Queloz 1995). The reduction procedure includes optimum extraction of the orders and flat-fielding achieved through a tungstene lamp exposure, wavelength calibration with a thorium lamp exposure, and removal of cosmic rays. Spectra are resampled every $0.03 \AA$ and corrected for the ELODIE transmission function. Cross-correlation functions using the CORAVEL technique are also automatically computed.

Spectroscopic observations were also carried out at La Silla using the $1.52 \mathrm{~m}$ ESO telescope with the FEROS spectrograph (Kaufer et al. 1998, 2000). The mean resolution of the spectrograph is $\lambda / \Delta \lambda \approx 48000$, the spectral coverage is from $3500 \AA$ to $9200 \AA$ and the exposure times varied from 45 to $60 \mathrm{~min}$. The reduction was automatically performed on-line by the FEROS routines, which include flatfielding, background subtraction, removal of cosmic rays, wavelength calibration and barycentric correction. Radial velocity corrections are applied and all the data shown are in the stellar rest frame. The spectra are not flux calibrated, so each spectrum has been continuum normalized.

Some observations were carried out at Lick Observatory, using either the $3 \mathrm{~m}$ Shane reflector or the $0.6 \mathrm{~m}$ Coudé Auxiliary Telescope (CAT) to feed the Hamilton Echelle Spectrograph (Vogt 1987) coupled to a FORD $2048 \times$ 2048 CCD. We recorded $\sim 92$ orders covering the optical spectrum from $\sim 3900 \AA$ to $\sim 8900 \AA$. The mean resolution of the spectra is $\lambda / \Delta \lambda \approx 48000$, and the exposure times varied 
from 45 to $75 \mathrm{~min}$, depending on the telescope used. The reduction was performed in a standard way described by Valenti (1994) which includes flatfielding with an incandescent lamp exposure, background subtraction, and cosmic ray removal. Wavelength calibration is made by observing a thorium-argon comparison lamp and performing a 2D solution to the position of the thorium lines as a function of order and column number. Radial and barycentric velocity corrections have been applied, and all the data shown here are in the stellar rest frame.

Spectra were also obtained with the SOFIN echelle spectrograph (Tuominen et al. 1999) at the $2.56 \mathrm{~m}$ Nordic Optical Telescope (NOT). The 3rd camera was used, which provides a spectral resolution of about $12 \mathrm{~km} \mathrm{~s}^{-1}(R=26000)$. The exposure time was $60 \mathrm{~min}$. The useful spectral range was from 4800 to $9400 \AA$ with some gaps in the red. The CCD images of the echelle spectra were reduced with the $4 \mathrm{~A}$ software package (Ilyin 2000). The standard procedure involves bias subtraction, correction for the flat field, scattered light subtraction with the aid of 2D smoothing splines, elimination of cosmic spikes and correction for vignetting function. The wavelength calibration was done with a Th-Ar comparison spectrum.

Additional observation were carried out with the cross-dispersed Coudé Echelle spectrograph of the 2-mAlfred-Jensch telescope of the Thüringer Landessternwarte Tautenburg on 6 nights between Nov. 27 and Dec. 21, 1999. A $2.0^{\prime \prime}$ slit was used together with the $1 \mathrm{k} \times 1 \mathrm{k}$ Tektronix CCD yielding a spectral resolution of about $\lambda / \Delta \lambda 35000$ over the wavelength range from 4630 to $7370 \AA$. Standard IRAF routines were used to flat-field and wavelength calibrate the spectra.

\section{Results}

\subsection{Photometry}

AA Tau's $V$-band light curve recorded over 150 days is shown in Fig. 1 (top panel). During these 5 months, the maximum brightness level was roughly constant at $V \sim 12.4$ and the photometric amplitude reached up to $1.0 \mathrm{mag}$. In the following, we mostly concentrate on the part of the light curve which has been the most heavily sampled by multi-site observations from JD 2451508 to 549 , which is also when simultaneous spectroscopic observations were obtained.

An enlargement of this section of the light curve is shown in Fig. 1 (middle panel). The photometric variations are nearly continously sampled over more than 3 weeks (from JD 508 to 533) and reveal large-scale brightness fluctuations occurring on a time scale of a few days with a maximum amplitude of 1.0 mag. This light curve bears some resemblance with the one we obtained in 1995 from a previous multi-site campaign (see Fig. 1 in Bouvier et al. 1999, hereafter B99). The maximum brightness level in the $V$-band is the same as in 1995 and is interrupted by luminosity dips lasting several days. Qualitatively similar dips were observed in 1995 with, however, a larger photometric amplitude (1.6 mag in 1995 compared to $1.0 \mathrm{mag}$ in 1999).

Another similarity between the 1995 and 1999 light curves is the $(B-V)$ color which exhibits little change as the system's brightness varies, except for a few transient flaringlike episodes. This is shown in Fig. 1 (bottom panel) where the $(B-V)$ light curve is seen to remain essentially flat within about 0.1 mag while the star's brightness varies by more than one magnitude in the $V$-band. A few short-term episodes with an amplitude of about 0.2 mag appear on JD 512/513 and 516 where the system suddenly turns bluer and on JD 529 when it turns redder on a timescale of a few hours. The small amplitude $(B-V)$ light curve which contrasts with the large luminosity variations had already been reported in the 1995 light curve, as well as the occurrence of transient blueing episodes (see B99, Fig. 2).

The photometric variations in the $B, R$, and $I$ filters are similar to those observed in the $V$-filter. Figure 2 illustrates the observed correlation between the photometric variations in the various filters. The $U$-band measurements are affected by large photometric errors (of order of $0.2 \mathrm{mag}$ due to the system's faintness at this wavelength) and will not be considered further. The slope of the linear least square fit to the observed correlations is given in each panel of Fig. 2 together with the fit rms, and the slope expected from interstellar reddening ("IS slope") is indicated. The correlation is particularly tight for the $V$ and $R$ filters. The $B$ vs. $V$ diagram exhibits a well-defined upper envelope with some points "dropping" from this envelope, which corresponds to the blueing episodes mentionned above. A noticeable feature of the $I$ vs. $V$ diagram is the apparent change of slope at $V \sim 12.8$.

A complementary representation of the color variations of the system is given in Fig. 3 where the $(B-V),(V-R)$ and $(V-I)$ colors are plotted against $V$ magnitude. With the exception of the blueing episodes, the near constancy within $0.1 \mathrm{mag}$ of the $(B-V)$ color is recovered in the $(V, B-V)$ diagram. The upper envelope of the points in this diagram suggests a possible trend for the system to become slightly redder at the lowest brightness levels, though with a much lower reddening slope than expected from insterstellar extinction. The average $(B-V)$ color of the system is $\sim 1.42$ in the 1999's light curve, while it was $\sim 1.25$ in 1995 , and the average $(V-R)$ color has remained the same between the two epochs. This probably indicates a slightly lower accretion rate in 1999 than in 1995, thus decreasing the blue excess and yielding a slightly red$\operatorname{der}(B-V)$ color without afecting much $(V-R)$.

The $(V, V-R)$ and $(V, V-I)$ diagrams indicate that the system gets redder when fainter up to $V \sim 12.8$, with a slope similar to that expected for interstellar extinction. Past this point, however, as the system's brightness further decreases the colors appear to saturate at a nearly constant value. An interesting feature of the $(V, V-I)$ diagram is the evidence for two parallel tracks around $V \sim 12.6$, where the system appears to oscillate between two $(V-I)$ color states. This phenomenon occurs within a few hours on JD 511 at the start of a large luminosity dip and will be discussed further below.

Overall, the luminosity and color variations observed in AA Tau in 1999 are qualitatively similar to those reported by B99 for the 1995 light curve. This leads us to believe that the dominant sources of photometric variability have not changed between the two epochs. A major difference, however, is that in 1995 the photometric variations were quasi-periodic on a 


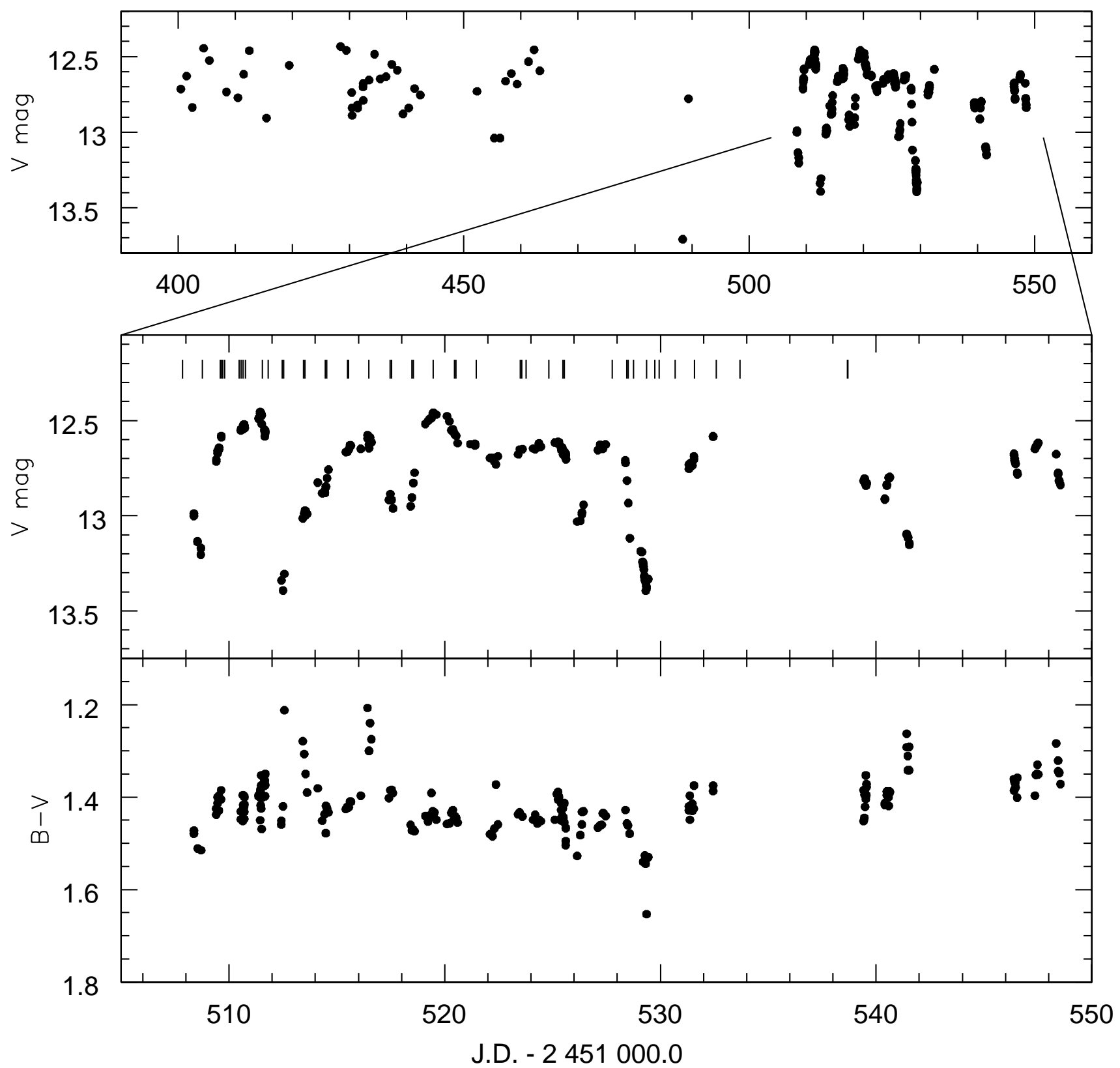

Fig. 1. AA Tau light curve. The numbers on the $x$-axis are the reduced Julian dates JD-2451000. Top: Full $V$-band light-curve. Middle: $V$-band light curve from JD 505 to 550; the vertical lines above the light curve indicate the dates of spectroscopic observations. Bottom: $B-V$ band light curve.

timescale of $\sim 8.3-8.6$ days. A periodogram analysis of the 1999's light curve reveals no significant period. Both the periodogram analysis (Scargle 1982) and the string-length method (Dworetsky 1983) suggest a best period of order of 16.5 days, but with a low confidence level. Photometric periods previously reported for AA Tau range from 8.2 days (Vrba et al. 1989; Shevchenko et al. 1991) to 8.3-8.6 days (B99). A periodogram analysis of AA Tau $V$-band light curve over 14 years, from 1987 to 2000, built from Mt Maidanak data also suggests a period of 8.1867 days (Grankin, priv. comm.). Additional support to the existence a $8.2 \mathrm{~d}$ period in the system is reported in the next section where we show that the radial velocity of the star appears to smoothly vary over this period.
Figure 4 shows AA Tau's $V$-band light curve with an illustration of the expected photometric variations assuming a $8.2 \mathrm{~d}$ period. The variations observed from JD 511 to 519 have been replicated on the rest of the light curve assuming a $8.2 \mathrm{~d}$ period. Several brightness maxima and minima appear to occur repeatedly on this timescale. This is the case for two main maxima (labelled A, B) and possibly a third one (C) at JD 511, 519, and 528 as well as for two secondary maxima $\left(\mathrm{A}^{\prime}, \mathrm{B}^{\prime}\right)$ and possibly a third one $\left(\mathrm{C}^{\prime}\right)$ on JD $\sim 516,524$, and 532. The two deepest minima $(\mathrm{a}, \mathrm{c})$ are separated by $\sim 17$ days, i.e., about twice the assumed period, and two secondary minima $\left(a^{\prime}, b^{\prime}\right)$ are in phase with the $8.2 \mathrm{~d}$ period. Clearly, the phase coherence is lost prior to JD 511 and after JD 535. Hence, even though the light 

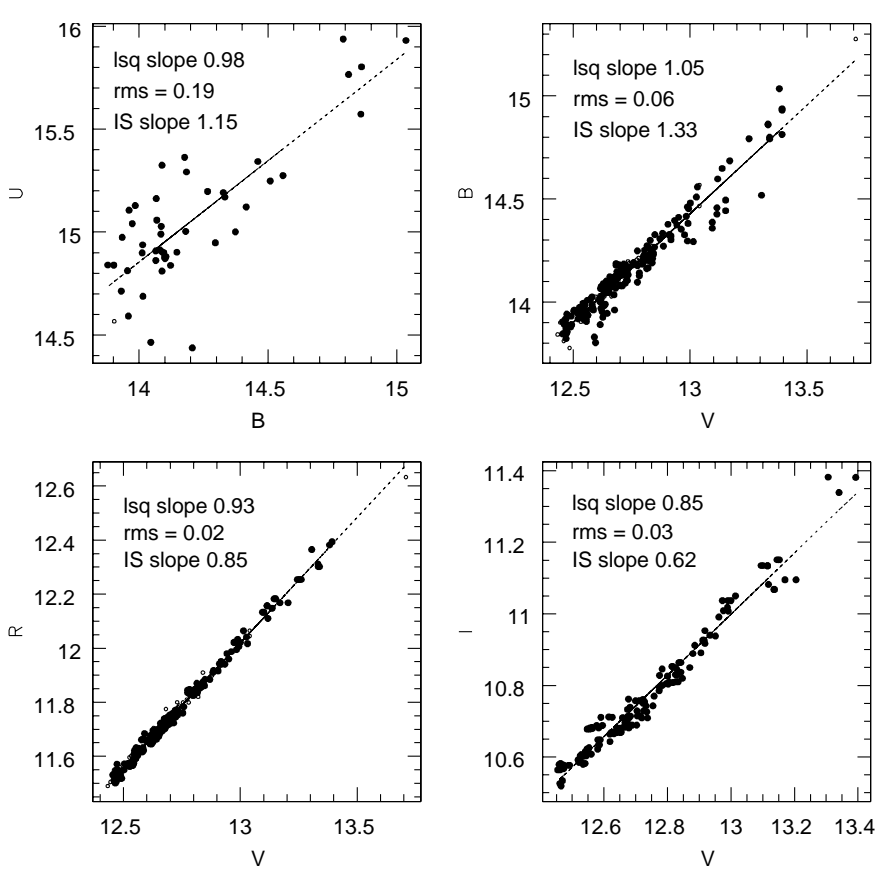

Fig. 2. Correlated brightness variations in $U B V R I$ filters. Small open dots: JD $\leq 500$, large filled dots: JD 505-550. Least-square fits to the observed correlations are shown as a solid line. The slope and rms of the fit are given in each panel, as well as the expected slope for an interstellar reddening law ("IS slope", Savage \& Mathis 1979).

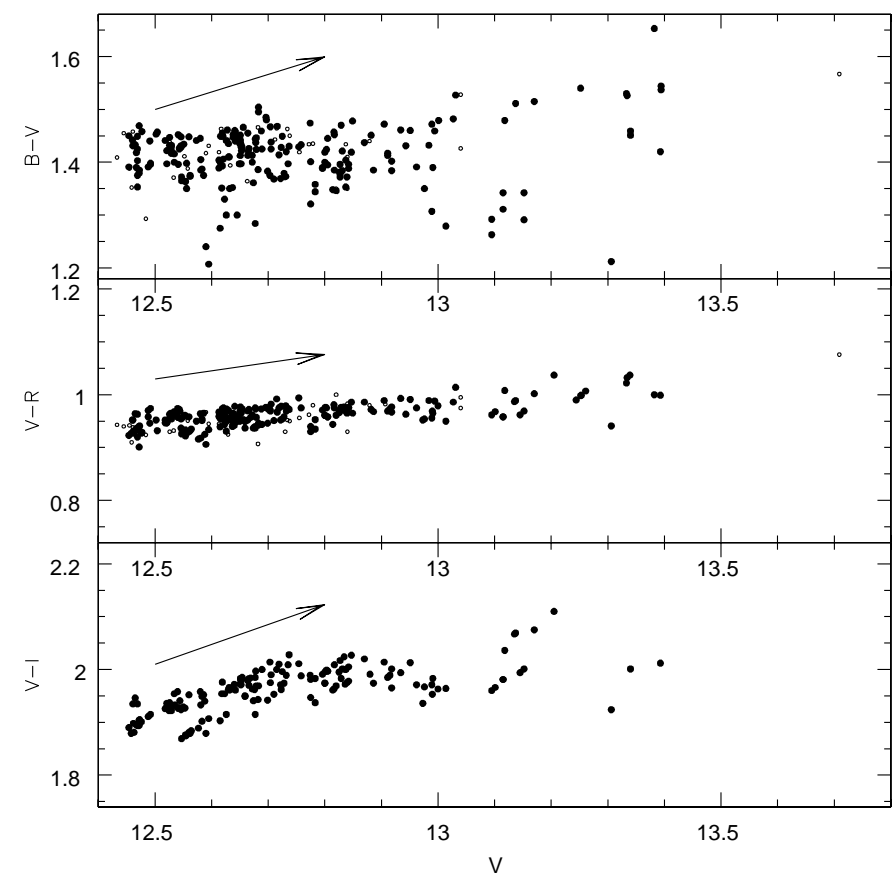

Fig. 3. Color-magnitude diagrams. The amplitude of the vertical scale is the same in each panel. Reddening vectors are illustrated for $A_{V}=$ 0.3 mag. Small open dots: JD $\leq 500$, large filled dots: JD 505-550.

curve is not periodic, we do find evidence for a characteristic timescale for the photometric variations which is consistent with the period reported previously by several authors.

The major discrepancy between the expected $8.2 \mathrm{~d}$ period and the observed light curve is the absence of a deep minimum

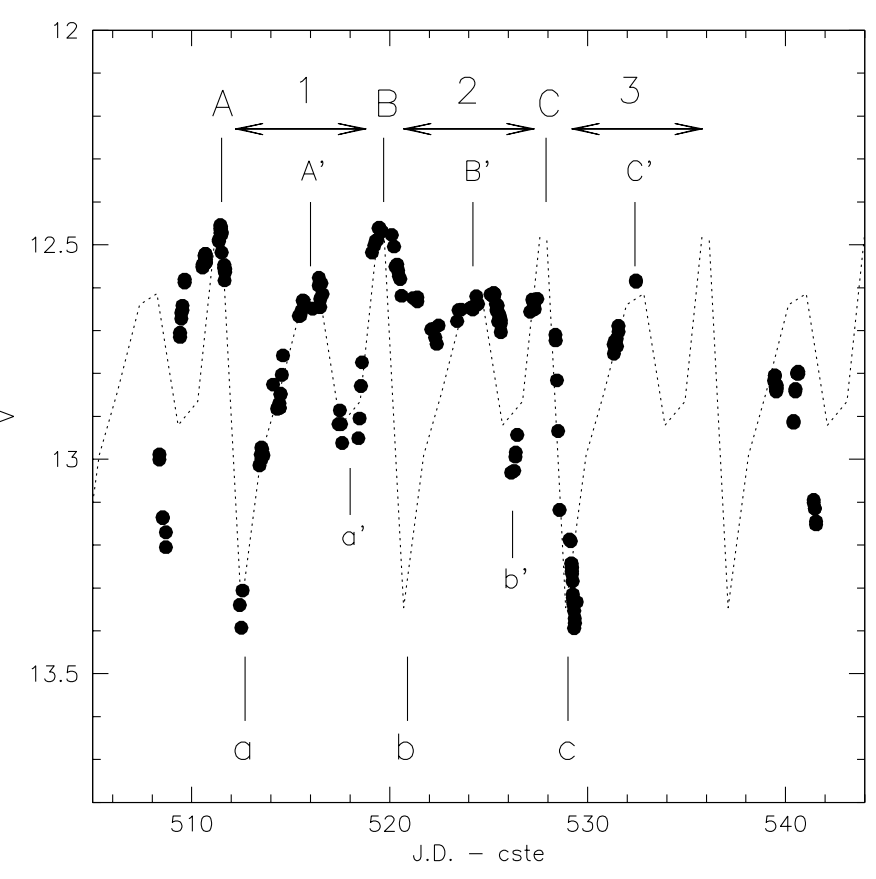

Fig. 4. AA Tau's $V$-band light curve. Part of the light curve between JD 511.5 and 519.5 ("cycle 1") has been replicated assuming a period of 8.2 days (dotted line). The various brightness minima and maxima are labelled with letters (see text).

around JD 521 (labelled "b" in Fig. 4). The flux of the system does decrease around this phase but only by about $0.2 \mathrm{mag}$, i.e., much less than during the two deep minima observed 8.2 days before and after this date, respectively. In the following, we will refer to this part of the light curve between JD $=519$ and JD = 525 as the "photometric plateau". Even more intringuing is the similar shape and depth of the two deep minima on JD 512 and 529, both being asymmetric with a rapid flux decrease and a more gradual return to maximum brightness. Assuming that the $8.2 \mathrm{~d}$ period is intrinsic to the system and probably reflects the rotational period of the star, the light curve suggests that one of the major sources of photometric variability has disappeared for one cycle but was restored on the next one. We show in the next section that a similar conclusion is reached from the analysis of the spectroscopic data.

\subsection{Spectroscopy}

AA Tau has been previously classified as a K7 dwarf (Kenyon \& Hartmann 1995). Its spectrum is that of a moderately active classical T Tauri star which exhibits clear photospheric lines and a few major emission lines, e.g. $E W(\mathrm{H} \alpha) \simeq 10-20 \AA$. The Balmer lines are characterized by the presence of a deep central absorption feature in the emission profiles (e.g. Edwards et al. 1994).

We discuss below the analysis of the 54 high resolution spectra of AA Tau obtained during the campaign. The projected rotational velocity of the star was measured from a correlation analysis of the photospheric spectrum. Veiling was measured on the 33 ELODIE and FEROS spectra from the comparison with a $\mathrm{K} 7$ template. The $S / N$ ratio of all spectra was high 
enough to investigate both the shape and the flux variations of the emission line profiles, most notably $\mathrm{H} \alpha, \mathrm{H} \beta$, and $\mathrm{He}$.

\subsubsection{Photospheric lines}

We first derive estimates of the AA Tau projected rotational velocity from 20 OHP spectra using the cross-correlation functions automatically computed with the ELODIE spectrograph and the calibration relation given in Queloz et al. (1998) for slow rotators. The derived vsini values range from 10.09 to $12.34 \mathrm{~km} \mathrm{~s}^{-1}$ with an average of $11.3 \mathrm{~km} \mathrm{~s}^{-1}\left(\mathrm{rms}: 0.7 \mathrm{~km} \mathrm{~s}^{-1}\right)$. This value fully agrees with the $v \sin i$ of $11.4 \mathrm{~km} \mathrm{~s}^{-1}$ computed by Hartmann \& Stauffer (1989).

We compute the veiling, defined as the ratio of continuum excess flux over photospheric flux, using a $\chi^{2}$ fit method on five spectral intervals typically $50 \AA$-wide located between 5500 and $6500 \AA$ and centered at: 5450, 5600, 5860, 6110 and $6420 \AA$ (see Fig. 5). We exclude from the fit the strong photospheric lines of $\mathrm{NaD}$ and $\mathrm{CaI} 6122 \AA$ which Stout-Batalha et al. (2000) have shown could be enhanced by the accretion process. We perform veiling calculation on the 33 FEROS and ELODIE spectra alone. Both have comparable high spectral resolution, sufficient signal to noise $(S / N)$ on the continuum level and sample the whole duration of the spectroscopic observations. For each data set, we compute the veiling relative to a reference AA Tau spectrum observed in the same instrumental configuration (an average of three spectra observed on JD 519.47, $520.45,520.52)$. The absolute veiling of this reference spectrum is then calibrated using the template weak line $\mathrm{T}$ Tauri star V819 Tau observed with ELODIE. V819 Tau (SpT = K7V) appears to be a very good spectral match to AA Tau (Fig. 5). Uncertainties associated with the relative variations of veiling, estimated from the rms of the five individual measurements, range from 0.01 to 0.05 . An additional uncertainty of typically 0.1 is however present on the absolute level of veiling.

The derived veiling values are low, ranging from undetectable to 0.3 , and do not correlate with the photometry (see Fig. 6 top panel, open circles). The main source of photometric variations is therefore not related to the continuum excess flux. Two main increases in veiling, lasting typically 3-4 days, occur at JD $=513.5$ and $\mathrm{JD}=516.5$ during respectively the egress phase of the first large photometric dip and just before the following small photometric dip (see Fig. 10). The veiling also increases at the very beginning and towards the end of the sampled light curve. During the photometric "plateau" (JD 519$525)$ the veiling is extremely weak. We observe a strong correlation of veiling with $(B-V)$ color and He I line flux (see Figs. 6 and 11). We also combine the measured veiling values with the $V$-band light curve to derive both the underlying "deveiled" photospheric flux and the flux of the continuum excess in the $V$ band (Fig. 6). We included in Fig. 6 only points where the photometry could be safely interpolated at the time of the spectroscopic observations. The excess flux variation closely follows the veiling one, with some scatter at high veiling values which will be discussed below.

We also compute, in the same wavelength intervals used to calculated the veiling, the radial velocity of photospheric lines by cross-correlation with the template spectrum, with a typical accuracy of $500 \mathrm{~m} \mathrm{~s}^{-1}$ (both our observing procedure and the correlation algorithm were not optimized for precise radial velocity measurements).

From the combined ELODIE and FEROS spectra (33), we measure an average heliocentric radial velocity of $17.1 \mathrm{~km} \mathrm{~s}^{-1}$ (rms of $0.9 \mathrm{~km} \mathrm{~s}^{-1}$ ) consistent with the previous derivation of $16.1 \pm 2.1 \mathrm{~km} \mathrm{~s}^{-1}$ (Hartmann et al. 1986). The amplitude of variation of the photospheric radial velocity is small $\left(\sim 2 \mathrm{~km} \mathrm{~s}^{-1}\right)$ but significantly larger than the estimated measurement errors $\left(\simeq 0.5 \mathrm{~km} \mathrm{~s}^{-1}\right)$. A string length analysis of the photospheric radial velocity variations yielded a most likely period of 8.288 days which is in agreement with the long term variability period found with the photometry. Figure 7 displays the photospheric radial velocity curve folded in phase with this period

\subsubsection{Survey of emisson line profiles}

A sample of the various residual line profiles discussed in the following sections is shown in Fig. 8. Residual profiles were obtained by continuum normalizing the AA Tau spectra and subtracting the normalized and veiled K7 template star (V819 Tau) used in the veiling measurements. We do not have veiling measurements for all spectra due to the low $S / N$ of some of the observations and in order to compute the residual profiles we used the nearest available veiling value. Since the observations are taken very close to each other and the highest measured veiling value is only about 0.3 , we should not be using unreasonable values. We note in Fig. 8 that the emission line profiles varied both in intensity and shape during our observations.

The $\mathrm{H} \alpha$ line presents double-peaked emission profiles, the blue emission peak generally more intense than the red one, resembling previously reported AA Tau profiles by Edwards et al. (1994), Muzerolle et al. (1998) and Alencar \& Basri (2000). The $\mathrm{H} \alpha$ profiles display both blueshifted and redshifted absorptions at low velocities in most of the observations as can be seen in Fig. 8. The blueshifted absorption is more intense than the redshifted absorption component and is also always present, while the redshifted absorption once totally disappeared from our observations for 2 days (Fig. 8d). The outer wings of the $\mathrm{H} \alpha$ profiles tend to be symmetric, but some profiles do present asymmetries in the outer red wing either as a lack of emission (most commonly, Figs. 8c, e) or as an extra red emission (Fig. 8f). The $\mathrm{H} \beta$ line profiles are also double-peaked but display most of the time a single absorption component that is centered or slightly blueshifted. A few $\mathrm{H} \beta$ profiles present an extra redshifted absorption at high velocities (Figs. 8c, e) and these tend to correspond to the asymmetric $\mathrm{H} \alpha$ spectra that show a lack of emission in the outer red wing. The He I line shows only a narrow component (NC) that is slightly redshifted. It is clearly asymmetric in some observations (Figs. 8a, c, h), with more emission in the red than in the blue side of the profile. Edwards et al. (1994) present AA Tau profiles of He I with broad and narrow components but at a much higher veiling value $(r=0.6)$ than we observed. The NaD lines (see 

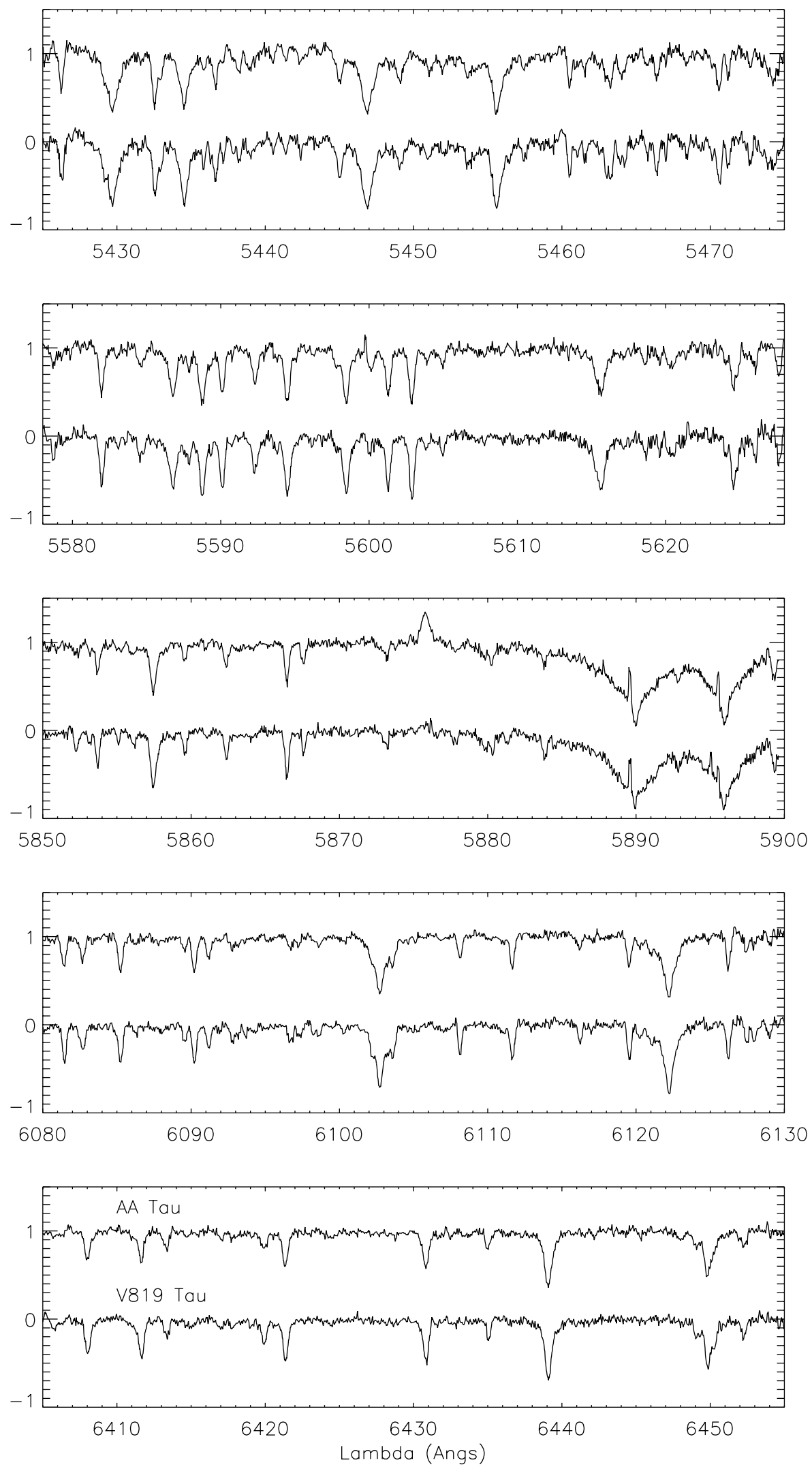

Fig. 5. Samples of an ELODIE spectrum of AA Tau (upper curve) obtained on JD 519.47. The spectrum of V819 Tau (lower curve), a K7 spectral template, is shown for comparison. In each spectral order the continuum has been normalized to unity and V819 Tau's spectrum has been shifted for clarity. The spectral orders shown in this figure were used to derive veiling (see text). The He I emission line ( 25876$)$ appears in emission in AA Tau's spectrum. 


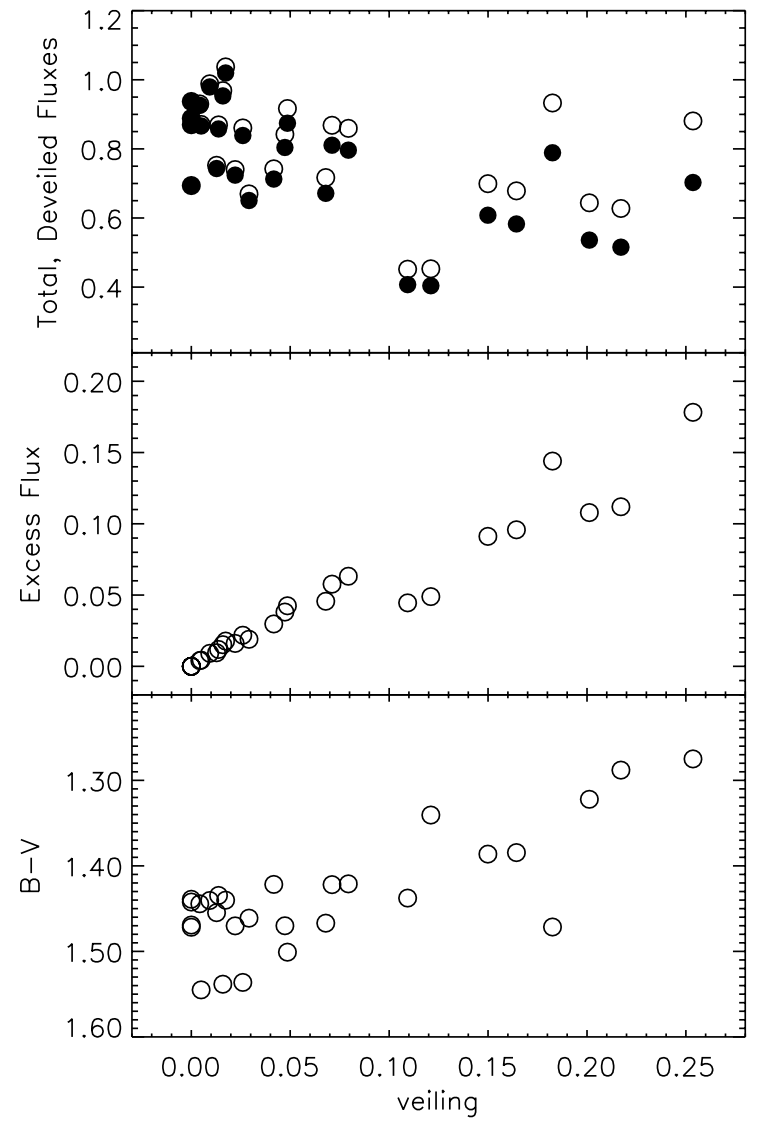

Fig. 6. Total flux in $V$-band (top panel open circles), de-veiled flux (top panel filled circles), excess flux (middle panel) and $B-V$ (lower panel) vs. veiling. The fluxes were interpolated on the veiling dates and are presented in arbitrary units.

Fig. 5) match very well the template spectrum and are basically photospheric.

\subsubsection{Variance profiles}

We show in Fig. 9 the average residual line profiles of $\mathrm{H} \alpha$ calculated with all the observations (left) and at different photometric epochs: during the deep minima (middle) and the photometric "plateau" (right). Also shown in the shaded area are the normalized variance profiles as defined by Johns \& Basri (1995b), which measure the amount of variability of each velocity bin in the line. The total $\mathrm{H} \alpha$ profile shows a rather uniform variability, the red wing presenting only a slightly more extended variability than the blue wing. The profiles taken during the photometric "plateau" and the deep minima are quite different from each other. The largest spectroscopic variations occur during the deep photometric minima. In contrast, the line profile does not vary much during the photometric "plateau" and its intensity is also much weaker. In agreement with the photometry, this behavior indicates that from JD $=518$ to 526 a major source of variability of the system disappeared.

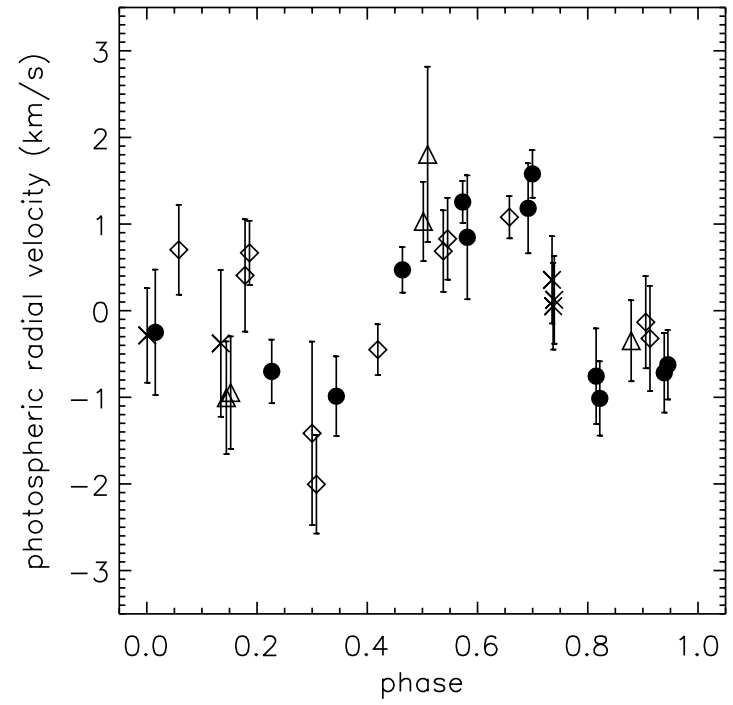

Fig. 7. Photospheric radial velocities folded in phase with a 8.288 day period. Different symbols represent successive cycles.

\subsubsection{Line fluxes and profile decomposition}

We measured the total equivalent widths of the $\mathrm{H} \alpha, \mathrm{H} \beta$ and $\mathrm{He} \mathrm{I}$ lines in order to compute line fluxes with the photometric measurements as $F(\mathrm{H} \alpha)=$ const. $\times E W(\mathrm{H} \alpha) \times 10^{-0.4 m_{R}}$, $F(\mathrm{He} \mathrm{I})=$ const. $\times E W(\mathrm{He} \mathrm{I}) \times 10^{-0.4 m_{V}}$ and $F(\mathrm{H} \beta)=$ const. $\times$ $E W(\mathrm{H} \beta) \times 10^{-0.4 m_{B}}$, where const. is an arbitrary constant and $m_{R}, m_{V}$ and $m_{B}$ are the $R, V$ and $B$-band magnitudes of the system, respectively. The photometric and spectroscopic measurements were not always simultaneous so we interpolated the light curves at the time of the spectroscopic observations in order to get the correct values for the magnitudes. No extrapolation was made. The line fluxes obtained are presented in Fig. 10. We looked for periodicities in the line flux variations using the Scargle (1982) periodogram estimator as modified by Horne \& Baliunas (1986) that is appropriate to handle irregularly spaced data and the string-length method (Dworetsky 1983). Both methods yielded similar results, showing best period detections around 16 or 8.5 days but with rather high false alarm probabilities (a few percent) of being created by chance.

We observe a strong correlation of veiling with the He I line flux (see Fig. 11). Two pairs of points however significantly depart from the veiling-HeI flux correlation at $\mathrm{JD}=512.5$ and 513.5, just after the minimum in the first large photometric dip. We come back to this in the discussion section. There is an indication of a weak correlation with $\mathrm{H} \beta$ line flux but nothing with $\mathrm{H} \alpha$. We looked for time-delayed correlations between the line fluxes and veiling and found that $\mathrm{H} \alpha$ presented a better correlation with veiling if its variations occured 1.08 days before the veiling variations (Fig. 11). The $\mathrm{H} \beta$ results showed the best correlation is obtained with a 0.44-day delay (Fig. 11) and the He I line is better correlated with the veiling with a time delay of 0.08 day (Fig. 11). The time lag between line flux and veiling variations is larger for lines that are formed farther away from the photosphere as predicted by the magnetospheric accretion scenario. 

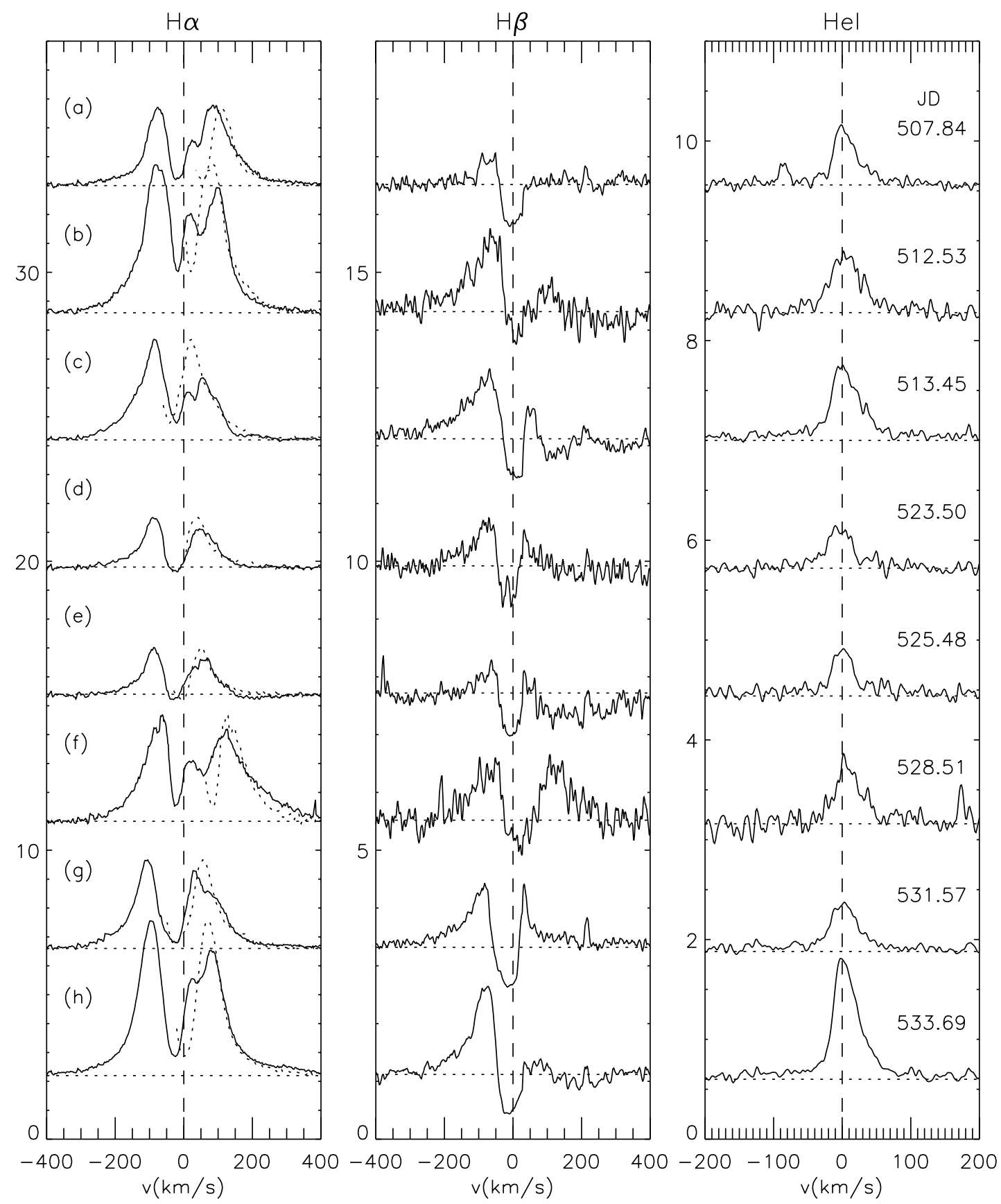

Fig. 8. Sample of residual line profiles. The profiles have been shifted for clarity. The vertical dashed lines are the spectral line center at the stellar rest frame and the horizontal dotted lines show the continuum level. The $\mathrm{H} \alpha$ blue wing has been reflected with respect to line center over the red wing. The numbers on the right are the dates of observation JD-2 451000.0 .

We calculated correlation matrices for the main emission lines in order to investigate how the profile variations are correlated across the line. Correlation matrices are 2D contour plots of linear correlation coefficients (see Johns \& Basri 1995b). The coefficients are calculated, in this work, correlating the time variation of each velocity bin of a spectral line with the time variation of all the other velocity bins of the same line or of a different line. Using all the observed $\mathrm{H} \alpha$ profiles, the outer blue and red wings correlated well with themselves but showed almost no correlation with each other (Fig. 12), which is rather unexpected since they are both thought to come from the high-velocity regions of the accretion flow. However, if we carefully select only the $\mathrm{H} \alpha$ spectra with symmetric red wings, taking away those that showed a lack or an excess of emission compared to the blue wing, the outer wings do correlate (Fig. 13). This indicates that in addition to the low velocity absorption in the red wing, there is also something going on at high velocities that affects the $\mathrm{H} \alpha$ profile and consequently its correlations. Although a redshifted absorption component is never clearly seen in the outer red wing of $\mathrm{H} \alpha$, it is probably present and sometimes can be seen in outer red wings of $\mathrm{H} \beta$ (see Fig. 8). Looking at the symmetric profile matrix (Fig. 13), 

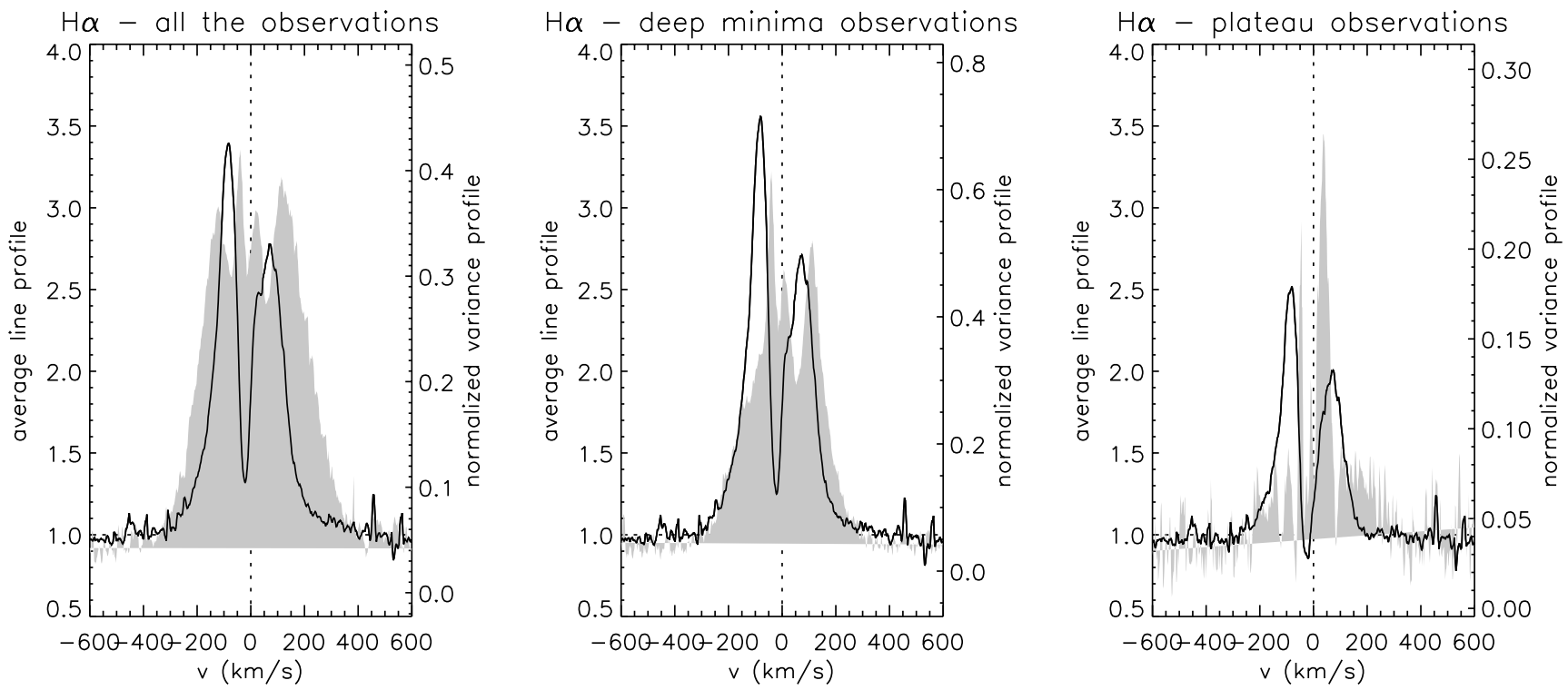

Fig. 9. Average $\mathrm{H} \alpha$ line profiles (solid lines) and variance profiles (grey shaded areas) calculated with residual spectra. Left: all the H $\alpha$ observations. Middle: the $\mathrm{H} \alpha$ observations taken during the deep photometric minima. Right: the observations taken during the photometric "plateau" (from $\mathrm{JD}=518$ to $\mathrm{JD}=526$ ).

the regions that do not correlate with the rest of the profile correspond to the blue and red low-velocity absorptions. We notice that the redshifted absorption at low velocities moves around much more accross the profile than the blueshifted one, thus creating a larger region with no correlation. No anti-correlated regions were found in the matrices, as also noticed Johns \& Basri (1995b) when they calculated the $\mathrm{H} \alpha$ correlation matrix of AA Tau.

The $\mathrm{H} \alpha$ and $\mathrm{H} \beta$ lines display more than one component with different characteristics and variability. In order to investigate the behavior of each line component separately we decomposed the $\mathrm{H} \alpha$ profiles using three Gaussians, corresponding to a centered emission, a blueshifted absorption and a redshifted absorption (see Fig. 14). We did not make any attempt to fit the high velocity redshifted absorption component that is conspicuous only in some $\mathrm{H} \beta$ profiles. The $\mathrm{H} \alpha$ decomposition is not always straightforward since there are two low-velocity absorption components superimposed on the main emission profile. The equivalent width of the absorption components are most of the time quite uncertain due to their proximity that makes it hard to disentangle them at our present resolution. The radial velocities of the three components however are well determined by the Gaussian decomposition. The radial velocity of the emission component is very well constrained by the profile wings and it surprisingly varied from $-45 \mathrm{~km} \mathrm{~s}^{-1}$ to $+30 \mathrm{~km} \mathrm{~s}^{-1}$. The radial velocity of the blueshifted absorption component varied from $-38 \mathrm{~km} \mathrm{~s}^{-1}$ to $-5 \mathrm{~km} \mathrm{~s}^{-1}$ and that of the redshifted absorption component from $2 \mathrm{~km} \mathrm{~s}^{-1}$ up to $70 \mathrm{~km} \mathrm{~s}^{-1}$.

We found a very good correlation between the radial velocities of the $\mathrm{H} \alpha$ blue and red absorption components (Fig. 15). Except for the three points at the uppermost left side of the plot (JD 529.5, 530.5, 531.5), the radial velocity of the red absorption component changes nearly twice as fast as the blue one, indicating that they are not driven by the same processes.
The blueshifted absorption component is thought to come from a wind, while in general redshifted absorption components are related to the accretion process. This result is therefore a piece of evidence of a correlation between accretion and outflow in AA Tau.

The $\mathrm{H} \beta$ line should have been easier to decompose than $\mathrm{H} \alpha$ but the low $S / N$ of our spectra in this region prevents a reliable decomposition most of the time. The He I line was decomposed with one Gaussian in emission and it presented small radial velocity variations, from $-4 \mathrm{~km} \mathrm{~s}^{-1}$ to $11 \mathrm{~km} \mathrm{~s}^{-1}$ (see Fig. 10).

\section{Discussion}

AA Tau's exhibited a more complex pattern of photometric variability in 1999 than previously observed in 1995. There are qualitative similarities between the two light curves which suggests the main source of variability has remained the same between the two epochs. There are, however, major differences as well. The 1999 light curve does not appear as periodic as it was in 1995: the latter light curve exhibited well defined, large amplitude luminosity dips on a quasi-periodic timescale of 8.2 days while the new light curve exhibits two smaller amplitude and asymmetric dips on this timescale and one of the major luminosity dips disappeared during one cycle. A rich pattern of variability is also seen in the emission line profiles and fluxes. Combining the photometric and spectroscopic results we attempt below to outline a global model for the origin of AA Tau's variability.

\subsection{Origin of the photometric variations}

The similarity of the light curve recorded for AA Tau in 1999 with that obtained in 1995 suggests that the same dominant mechanisms were reponsible for the observed photometric variability at the two epochs. B99 interpreted the photometric 

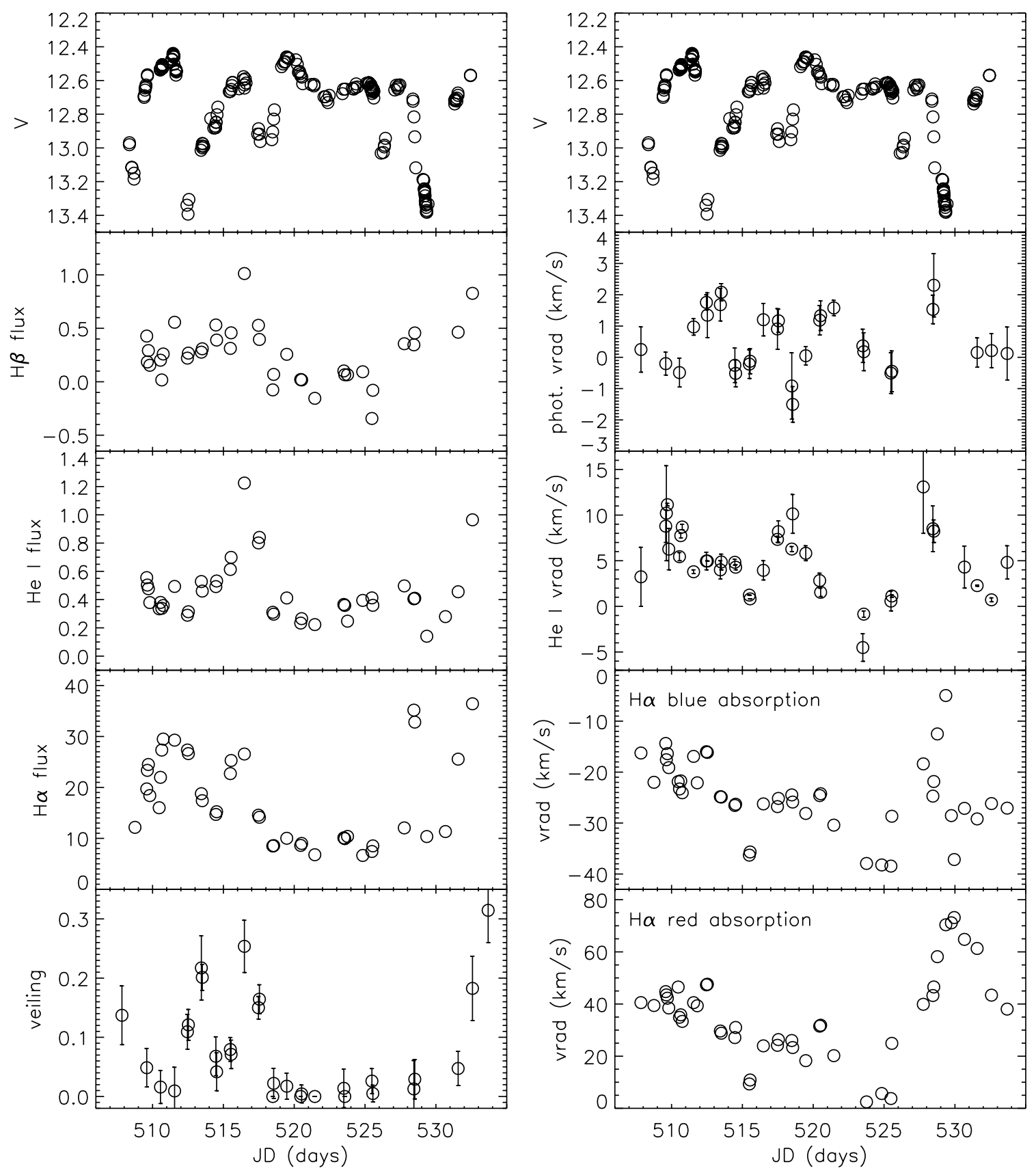

Fig. 10. $V$ magnitude, $\mathrm{H} \alpha, \mathrm{H} \beta$, He I line fluxes and veiling (left panel) and $V$ magnitude, photospheric radial velocity, He I radial velocity, and radial velocity of the blue and red absorption components in the $\mathrm{H} \alpha$ profile (right panel). The line fluxes are in arbitrary units.

behaviour of this system as resulting from the obscuration of the stellar photosphere by circumstellar material orbiting the star at Keplerian velocity. The opacity screen responsible for the eclipses was tentatively identified with the warped inner edge of AA Tau's circumstellar disk close to the corotation radius where it interacts with the star's inclined magnetosphere. This interpretation has subsequently gained support from the physical modelling of the response of a circumstellar disk to an inclined dipole as the models predict the development of a non axisymmetric warp at the inner disk edge (Terquem \& Papaloizou 2000; Lai 1999).

Arguments against alternative interpretations of AA Tau's light curve were given in B99. The new light curve and spectroscopic data provide additional ones that we summarize here briefly.

\subsubsection{A planetary mass companion?}

The detection of radial velocity variations with a period of 8.3 days and an amplitude of order $2 \mathrm{~km} \mathrm{~s}^{-1}$ (see Fig. 7) may point to the existence of a low-mass companion orbiting the star. B99 discussed this possibility before uncovering the periodic radial velocity variations. Using the newly derived amplitude of the radial velocity curve, we can now derive a maximum mass (assuming a circular orbit) of 20 Jupiter masses for the putative companion. However, it is unlikely that such 

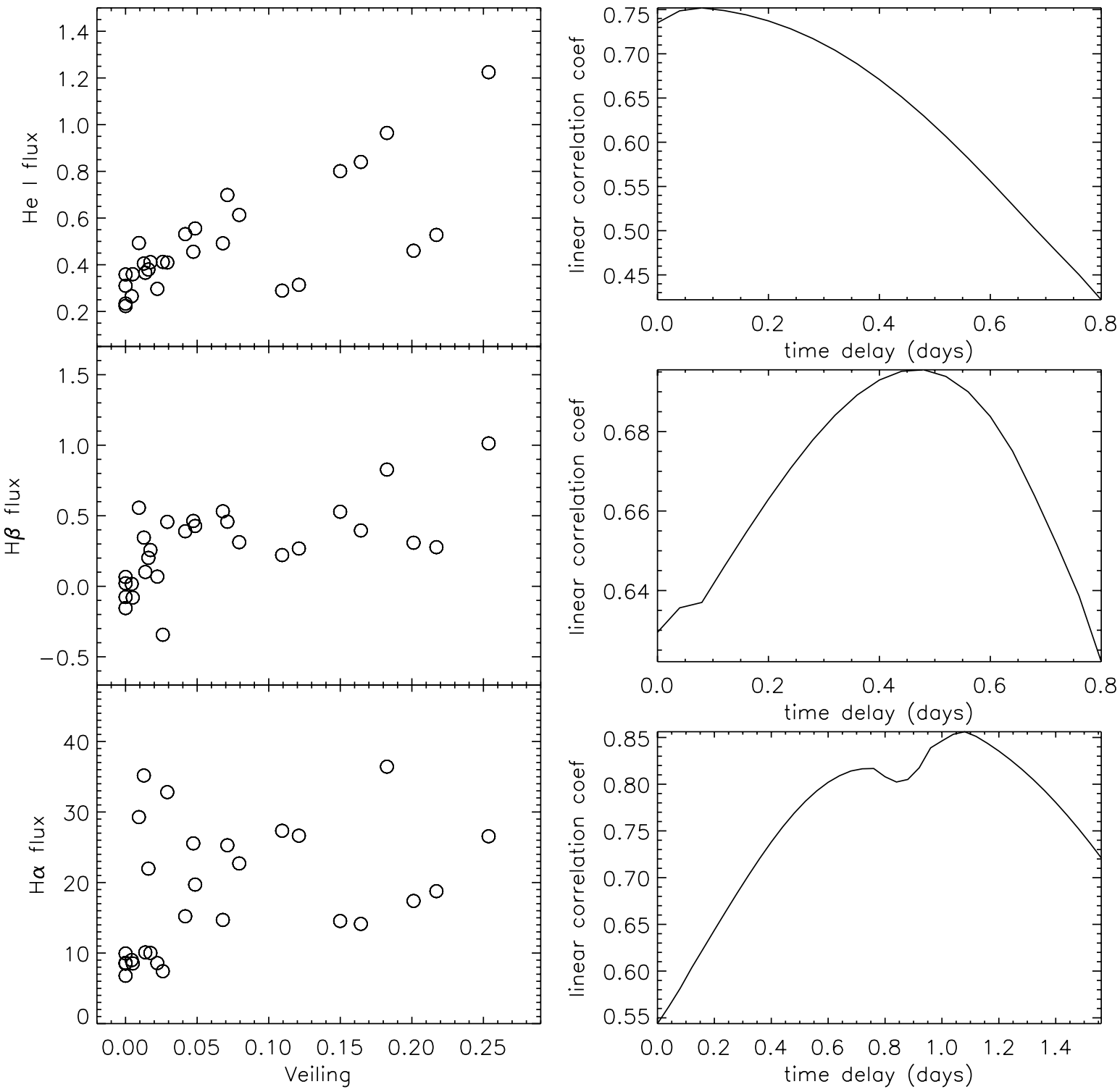

Fig. 11. Left panel: The $\mathrm{H} \alpha, \mathrm{H} \beta$ and $\mathrm{He} \mathrm{I}$ line fluxes are plotted against veiling. Right panel: Time delay between emission line flux and veiling variations which provides the best correlation between the two quantities (see text). From top to bottom: $\mathrm{He}$ I $(0.08 \mathrm{~d}), \mathrm{H} \beta(0.44 \mathrm{~d})$ and $\mathrm{H} \alpha(1.08 \mathrm{~d})$. A positive time delay means that the line flux varied before the veiling did.

an orbiting companion could be responsible for the observed photometric variability. The non-steady character of the photometric light curve and, in particular, the temporary disappearance of one of the major luminosity dips during one cycle, conflicts with the stability expected from the orbital motion of a substellar companion. In addition, the radial velocity variations are still present during the photometric plateau, i.e., when the large luminosity dip has desappeared (cf. Fig. 10). Hence, there seems to be no direct connection between the source of the large scale photometric variability and the source of the radial velocity variations.

Interpreting the radial velocity variations of AA Tau as reflex motion induced by an orbiting low mass companion would imply that the orbital period of the companion (8.3 d) is similar to the stellar rotational period $(8.2-8.5 \mathrm{~d})$. This could possibly result from the companion having experienced type II inward migration in the disk which stops at the inner disk edge (Lin et al. 1996). In AA Tau, the disk truncation radius lies near or at the corotation radius ( $0.08 \mathrm{AU}$, see $\mathrm{B} 99)$, thus leading to an orbital period at this radius similar to the stellar rotational period. In order to further investigate the possible existence of a substellar companion in close orbit around AA Tau, a new spectroscopic monitoring campaign is planned for the fall 2003 which will measure the star's radial velocity curve over several months.

\subsubsection{Surface spots}

Alternatively, cold surface spots may be responsible for the periodic variations of the radial velocity. The $8.3 \mathrm{~d}$ period of the radial velocity curve is consistent with the previously reported rotational period of AA Tau in the range 8.2-8.5 d. We used 


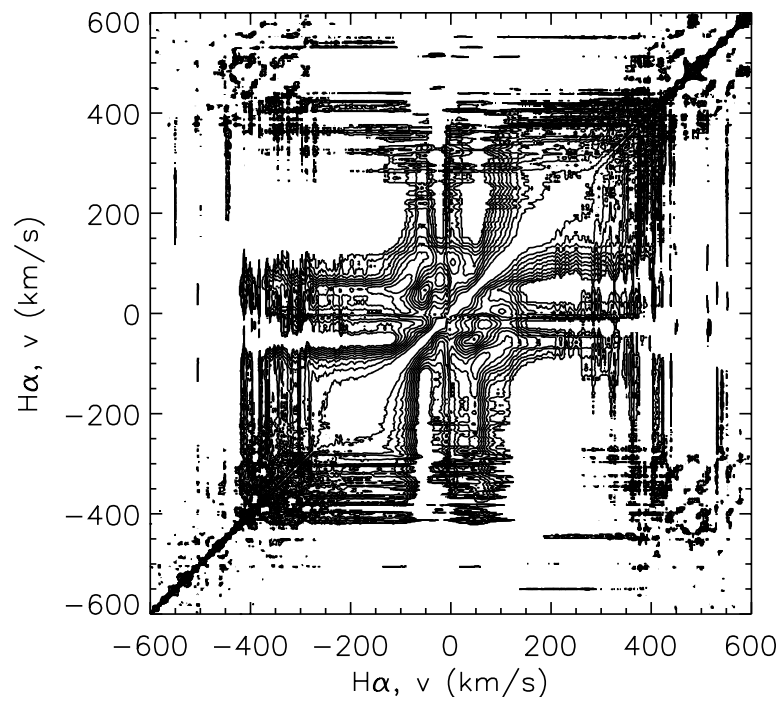

Fig. 12. $\mathrm{H} \alpha$ vs. $\mathrm{H} \alpha$ correlation matrix calculated with all the observed profiles.

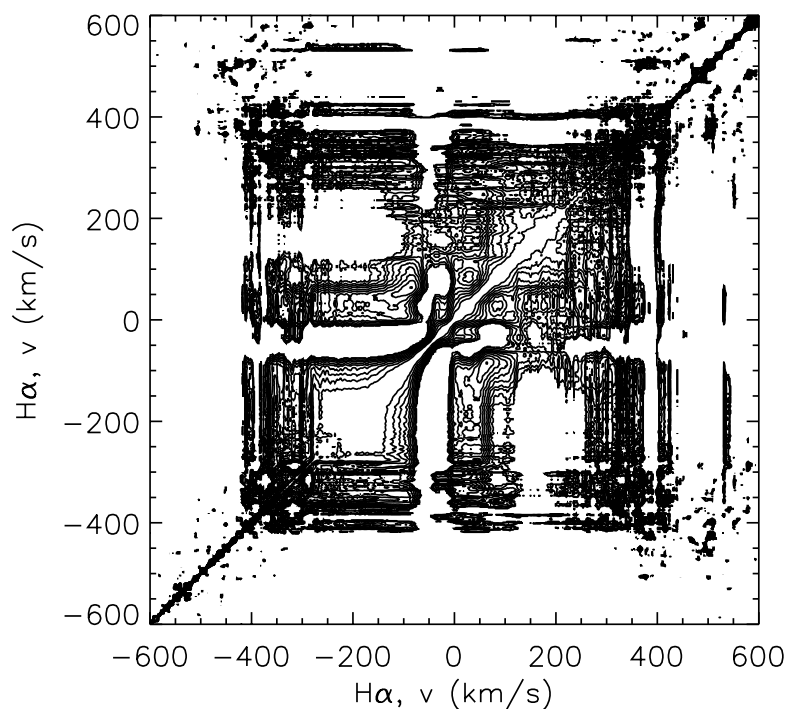

Fig. 13. $\mathrm{H} \alpha$ vs. $\mathrm{H} \alpha$ correlation matrix calculated without the profiles that presented asymmetric red wings.

Petrov et al.'s (2001) model to compute the radial velocity variations induced by a dark surface spot. We find that a $38^{\circ}$ radius spot located at a latitude of $55^{\circ}$ would produce a periodic modulation of the stellar radial velocity with an amplitude of $1.6 \mathrm{~km} \mathrm{~s}^{-1}$, slightly smaller than observed. Hence, spots of at least this size would be required to account for AA Tau's radial velocity amplitude. However, such a single circular spot would also produce a modulation of the stellar luminosity with an amplitude of about 0.26 mag in the $V$-band. According to the phase of AA Tau's radial velocity curve, the cold spot would be at maximum visibility around JD 515, 523 and 531. Brightness variations observed at these dates tend to be lower than those predicted by the model. Hence, we failed to find a spot model which consistently accounts for AA Tau's radial velocity and brightness variations.

Independently of the radial velocity variations, ascribing the large luminosity dips of AA Tau's light curve to
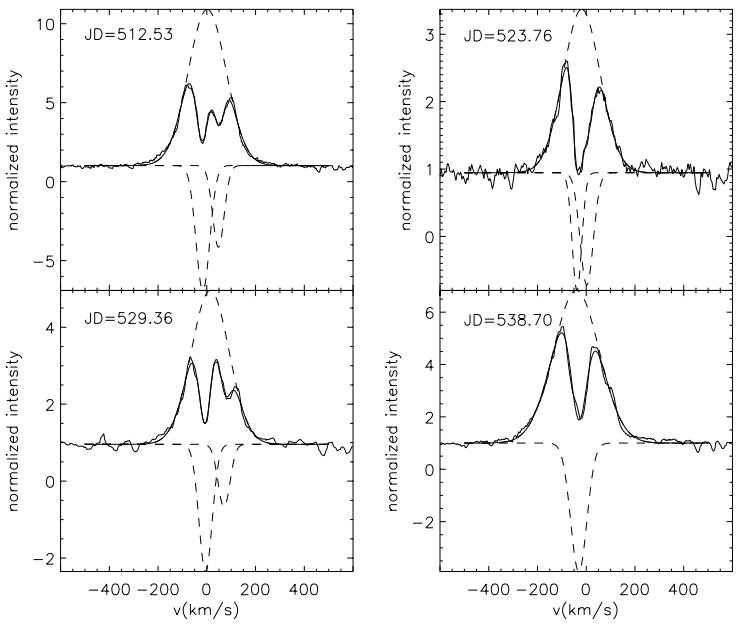

Fig. 14. Examples of the decomposition of the $\mathrm{H} \alpha$ line profile using three Gaussians. Thin solid line: observed spectrum, dashed lines: individual components, thick solid line: all components added together.

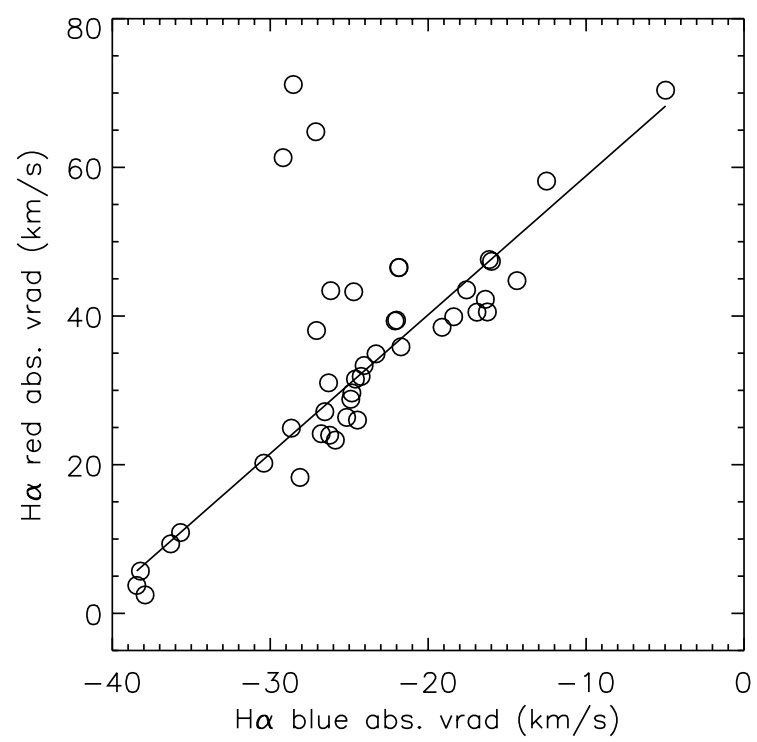

Fig. 15. Radial velocities of the $\mathrm{H} \alpha$ red and blue absorption components. A tight correlation is observed between the two quantities. The three data points that strongly depart from this correlation occurred on JD 529.5, 530.5 and 531.5.

photospheric cold spots would require a projected spot area covering at least $50 \%$ of the visible photosphere, i.e., a spot radius of about $45^{\circ}$ if circular. For a stellar rotation period of $8.2 \mathrm{~d}$ days, it would take at least a couple of days for such a large spot to go from totally invisible to fully visible, regardless of its shape. This is inconsistent with the sharp luminosity decrease observed in the light curve where the system dims by 1 mag on a timescale of a day or less. In addition, huge cold spots observed at the surface of WTTS are stable on a timescale of several weeks at least and up to several years (e.g. Petrov et al. 1994). The missing large luminosity dip around JD 522 in AA Tau's light curve would conflict with this expectation. One might assume that AA Tau's rotational period is $16.4 \mathrm{~d}$ instead of $8.2 \mathrm{~d}$ to circumvent this problem, but this is ruled out from $v \sin i=11.3 \pm 0.7 \mathrm{~km} \mathrm{~s}^{-1}$ (see above) which, with 
$R_{\star}=1.85 \pm 0.15 R_{\odot}(\mathrm{B} 99)$, yields $P=(8.29 \mathrm{~d}+/-1.2 \mathrm{~d}) \cdot \sin i$, i.e. a maximum rotational period of about 8.5 days for an edgeon system.

Hence, we believe that large luminosity dips cannot be due to cold surface spots. The smaller, secondary luminosity dips are also unlikely to be due to cold spots since their duration does not exceed 2 or 3 days, while the modulation by surface spots would be expected to produce at least 4-day wide dips for a stellar rotation period of 8.2 days and $\sin i \simeq 1$. Furthermore, the small dips exhibit the same color behaviour as the large ones (cf. Figs. 2, 3) which suggests a common cause.

Hot spots are also easily dismissed as a possibly dominant cause of AA Tau's photometric variability since one would then expect a correlation between the system brightness and veiling or excess flux due to the accretion shock. Such a correlation is not observed and the excess flux actually tends to be larger when the system is fainter (see Fig. 6).

\subsubsection{Variable circumstellar extinction}

Various aspects of AA Tau's photometric variations point to variable circumstellar extinction: the constant maximum brightness of the light curve over 150 days (see Fig. 1), the small amplitude of $(B-V)$ color changes as the system dims except for transient events (see Fig. 3), the duration of the dips, their sharp ingress phase and asymmetric shape, and the lack of a strictly periodic behaviour can all be accounted for by the partial occultation of the stellar photosphere by orbiting circumstellar material. Moreover, Ménard et al. (2003) reported increased polarisation levels during the faintening episodes, as expected from the obscuration of the photosphere by circumstellar dust. While other minor sources of photometric variability might be present as well, neither stellar spots nor the orbital motion of a companion could account for all these features simultaneously.

Another supporting evidence for circumstellar extinction comes for the color behaviour of the system. We found above that the $(B-V)$ color is not significantly affected during luminosity dips (apart from short timescale events discussed below) while in the $(V-R)$ and $(V-I)$ colors the system first reddens as it dims. Such a wavelength-dependent behaviour may result from extinction by dust particules slightly larger than the interstellar ones, producing opaque occultations at short wavelengths and interstellar-like reddening at longer ones. The change of the $(V-R)$ and $(V-I)$ color slopes as the system dims further (see Fig. 3) also suggests non uniform extinction properties of the occulting screen, which becomes more opaque as the occulation progresses.

Rapid color changes, best seen in the $(B-V)$ color, are found to occur at specific photometric phases. Two blueing episodes developped on a timescale of a few hours around JD 512 and 516, while a rapid reddening event occurred around JD 529. These events are associated to the luminosity dips, though they can occur either at the start or in the middle of the dips. The timescale associated with these events is comparable to the duration of transit of circumstellar material across the stellar photosphere (of order of 0.3 days if the occulting material is located at the corotation radius, cf. B99). The transient color excursions may thus be related to small-scale $(\leq 0.01 \mathrm{AU})$ structures in the absorbing material which would indicate that the occulting screen is somewhat clumpy.

A third pattern of color changes is observed on a timescale of a few hours. Figure 16 shows that the $(V, V-I)$ color diagram exhibits two parallel tracks separated by about $0.1 \mathrm{mag}$ in luminosity when the system is close to maximum brightness (Fig. 16b). The tracks themselves are roughly parallel to the reddening slope expected for extinction by small grains. On JD 511.5, at the very start of a large luminosity dip, the system was first located on the upper track, becoming redder when fainter. It then suddenly flipped onto the lower track at a slightly bluer color before starting to redden again as it further dimmed (Fig. 16c). The blueward transition from the upper to lower $(V, V-I)$ track took less than 3 hours on JD 511.5 and a qualitatively similar behaviour is observed in the $(V, V-R)$ and $(V, B-V)$ color diagrams. Other data points on the lower $(V, V-I)$ tracks are from JD 516.5. This rapid variability is observed close to maximum brightness, just before the occurrence of photometric dips, and indicates the sudden appearance of a source of blue continuum excess flux as the system starts to dim, possibly the accretion shock at the stellar surface. The strong increase in veiling observed on JD 512.5-513.5 and 516.5-517.5 (see Fig. 10), i.e., within a day after these blueing episodes supports this interpretation.

B99 identified the obscuring material as the warped inner edge of AA Tau's circumstellar disk close to the corotation radius, as the disk encounters the stellar magnetosphere. The characteristic timescale of 8.2 days is recovered in the new light curve, thus indicating that the circumstellar material is still located close to the corotation radius if in Keplerian rotation around the $\operatorname{star}\left(r_{\mathrm{co}} \simeq 8.8 R_{\star}\right.$, cf. B99). This is also consistent with the short duration of the ingress phases of the luminosity dips. However, the structure of the occulting material appears more complex than it was in 1995. The asymmetric shape of the major dips suggests a sharp leading edge for the occulting material and a smoother trailing edge. Also, the light curve indicates the occurrence of two occulation events per photometric cycle (cf. Fig. 17) separated by about $0.4-0.5$ in phase, suggestive of the presence of two occulting structures located at nearly opposite azimuths around the star. Finally, the depth of the eclipses is shallower in $1999(\sim 1 \mathrm{mag})$ than it was in 1995 ( 1.5 mag).

Because the AA Tau system is suspected to be seen nearly edge-on (see B99), the occulting material has to lie close to the equatorial plane and is thus likely associated with the circumstellar dusty disk. A smooth azimuthal warp of the inner disk, as suggested by B99 to account for the 1995 light curve, does not produce two occultations per orbital cycle. Terquem \& Papaloizou (2000) showed that the response of an accretion disk to an inclined stellar magnetosphere actually produces a warp with two vertical maxima on the upper side of the inner disk, located at opposite azimuths and having unequal amplitudes (see their Fig. 6). Such a warp can qualitatively account for the occurrence of two luminosity dips of unequal depth within one single orbital period of the inner disk edge. It is also worth noting that one of the magnetic configurations 

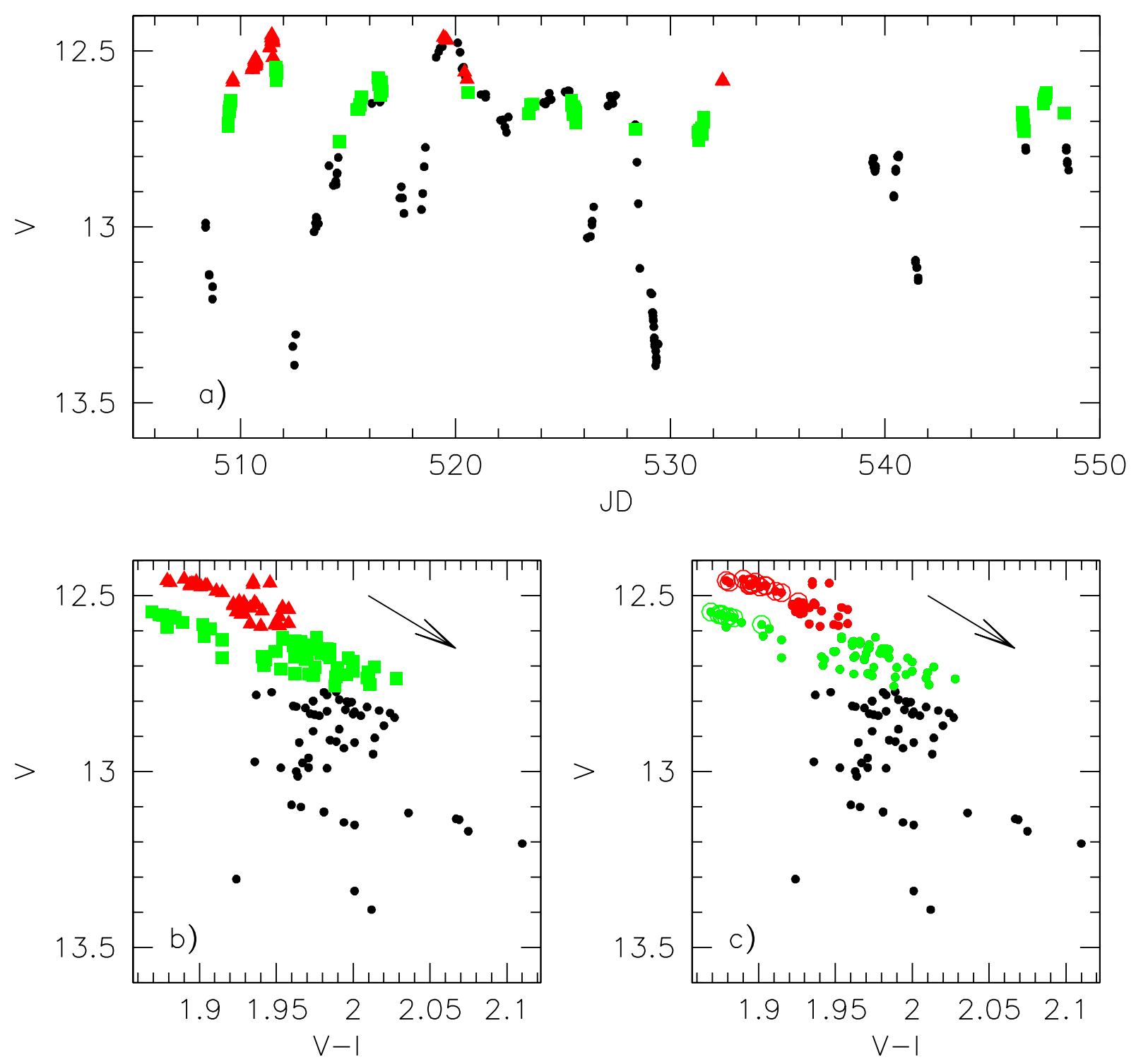

Fig. 16. Panel a) AA Tau $V$-band light curve. At specific dates, measurements are represented by large square and triangle symbols for reference with panel b). Panel b) $V$ versus $V-I$ diagram: close to maximum brightness, the system follows 2 parallel color tracks. Symbols are the same as in panel a). Panel c) $V$ versus $V-I$ diagram: the path followed in this diagram by the system on JD 511 is shown by large empty circles. As it dimmed, the object suddenly flipped from the upper to the lower color track. The arrow in panels b) and c) indicates the color slope expected from interstellar extinction.

explored by Terquem \& Papaloizou (2000) results in a vertical structure for the inner disk warp which mimics a trailing spiral pattern. Such a warp configuration would qualitatively account for the asymmetric shape of the main luminosity dips observed in AA Tau's light curve, with a sharp ingress phase and a slower return to maximum brightness. In any case, the differences between the '95 and '99 light curves indicate that the structure of the inner disk warp has changed between the two epochs.

\subsection{Validity of the magnetospheric accretion scenario}

The light curve presented here, as well as that obtained in 1995, appears to be best interpreted in terms of recurrent occulations of the central star by circumstellar material located at the warped inner edge of AA Tau's accretion disk. A likely origin for the inner disk warp is the response of the disk to an inclined stellar magnetosphere. B99 proposed a model for AA Tau where the accretion disk is disrupted by the stellar magnetosphere at a distance of $0.08 \mathrm{AU}$, resulting in circumstellar material being channelled onto the star along magnetic accretion columns and eventually hitting the stellar surface to produce two opposite accretion shocks located at intermediate latitudes (see their Fig. 10). This model was able to account for the major characteristics of the luminosity and color variations of the system in 1995.

We have now an opportunity to further test this model from the observed spectroscopic variations of the system along the photometric cycle. Several aspects of the AA Tau spectroscopic analysis seem to confirm the general scenario of magnetospheric accretion.

The veiling shows two peaks occuring per 8.2-day cycle and which last 3 to 4 days with the highest value around 0.3 

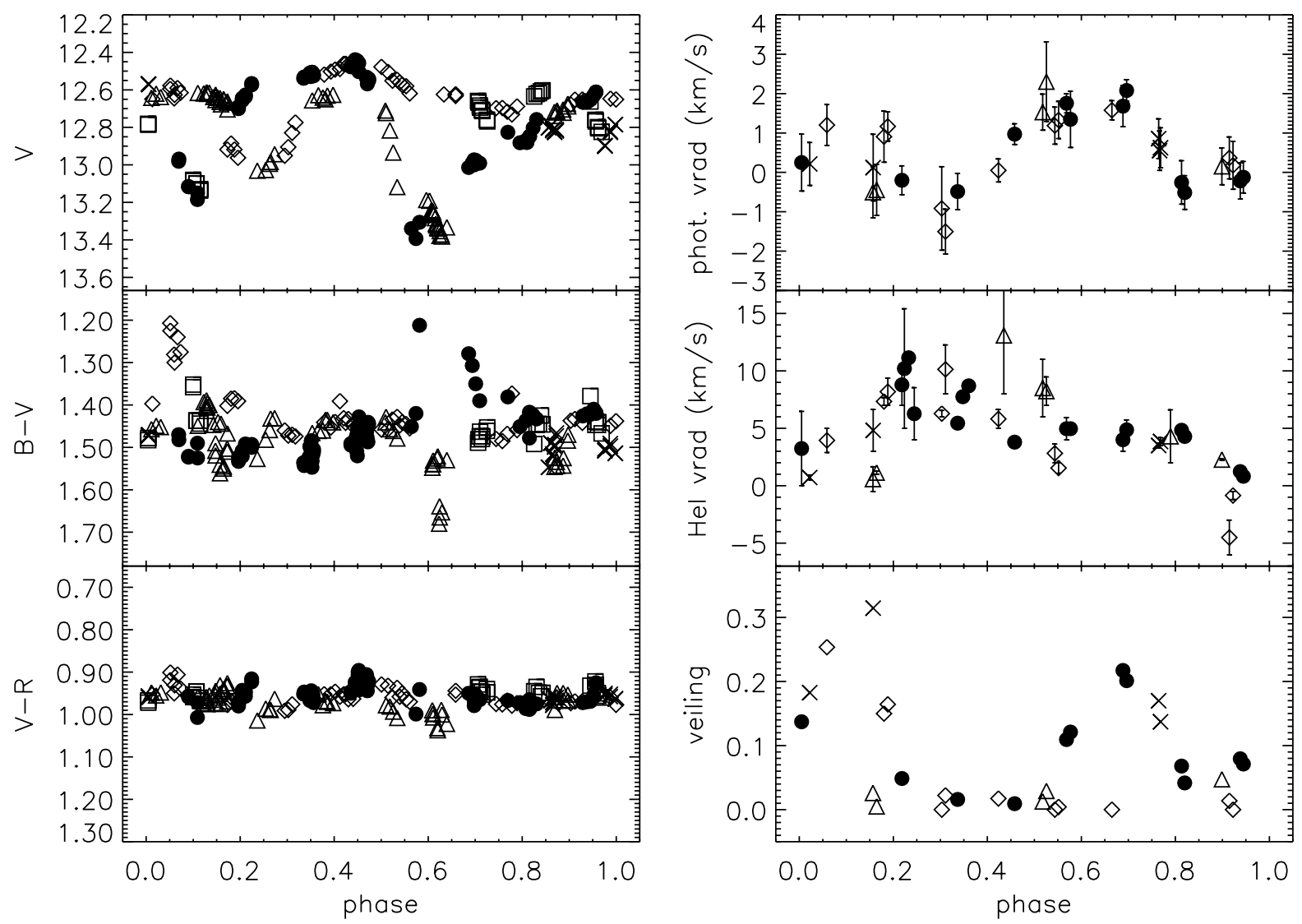

Fig. 17. $V$ magnitude, $B-V$ and $V-R$ (left), photospheric radial velocity, He I radial velocity and veiling (right) in phase with a $8.2 \mathrm{~d}$ period $\left(\mathrm{JD}_{0}=516\right)$. Different symbols represent different orbital cycles.

(see Fig. 17). The peaks suggest the presence of two rotationally modulated hot spots corresponding to the accretion shocks located around the magnetic poles at the stellar surface. The $B-V$ color of the system shows some correlation with veiling and at least the bluest $B-V$ values clearly correspond to the highest veiling values (Fig. 6). This confirms that blueing events are associated with veiling variations, both being produced by a hot source of continuum flux identified with the acretion shocks on the stellar surface.

One of the veiling maxima occur within one day of the center of the first deep photometric minima on JD 513 (see Fig. 10), which suggests that the hot spot faces the observer at this phase, in agreement with B99's geometrical model. Another veiling maximum occurs about 3-4 days later around JD 516-517, which would correspond to the maximum visibility of the second accretion shock, located on the opposite magnetic pole. Both episodes of increased veiling are preceeded by a sudden blueing of the system on JD 511.5 and 516.5 (see previous section) which may correspond to the appearance of the accretion spots on the stellar limb. Note that the veiling maxima seem to have disappeared during the photometric plateau (JD 519-525), which indicates that the mass accretion rate was much lower during that cycle. The third and highest veiling maximum is seen around JD 533 but the sparse sampling of both spectral and luminosity variations around this date prevent us from associating this event with a clear photometric pattern.
Figure 17 nevertheless indicates that this last veiling maximum is in phase with the one which occured on JD 516-517.

The veiling also strongly correlates with the He I line flux (Fig. 11) which is expected in magnetospheric accretion models, since the He I emission line is thought to form at the base of the accretion column close to the accretion shock. It is interesting to note that two pairs of He I flux measurements, on JD 512.5 and 513.5, are weaker than expected from the overall correlation. Since these dates correspond to the center of a large luminosity dip, this indicates that the He I emission region close to the stellar surface is partly occulted at the same time as the photosphere. It should be pointed out, however, that the apparent veiling is likely overestimated on these dates due to the occulation of the photosphere. Since veiling measures the ratio between the excess flux and the photospheric flux, the partial occultation of the photosphere artificially enhances veiling even if the excess flux remains constant. Thus, the 1 mag drop in the photospheric flux between JD 511 and 512 would result in an increase from 0.04 to 0.12 for the veiling. This only partly accounts for data points that strongly deviate from the average correlation between line flux and veiling. The same effect is seen in the correlation between the veiling and the continuum excess flux (see Fig. 6). As noted by B99, the sudden increase of veiling as the system dims actually reinforces the interpretation of AA Tau's photometric variations as being due to the occultation of the stellar photosphere. 
We see high velocity (150-300 $\mathrm{km} \mathrm{s}^{-1}$ ) redshifted absorption components in the wings of $\mathrm{H} \beta$ and $\mathrm{H} \alpha$. These occur most clearly at JD $=513.5$, simultaneously with the veiling peak, and at JD $=525.5$, with no increase of veiling associated to it. Redshifted absorptions at high velocity are usually associated to material free-falling onto the star along magnetospheric accretion columns. They ought to be observed when the hot spot is seen through the accretion columns along the line of sight. The simultaneous occurrence on JD 513.5 of a high velocity redshifted absorptions in the Balmer line profile and of maximum veiling is consistent with the accretion column being projected onto the hot spot facing the observer. This phase corresponds to the center a the large luminosity dip which lasts from JD 511.5 to JD 515.5. The other occurrence of a redshifted absorption component in the $\mathrm{H} \alpha$ and $\mathrm{H} \beta$ profiles is seen on JD 525.5. Even though veiling is weak on this date we argued above that this phase corresponds to the maximum visibility of the second accretion shock. The weak veiling and line fluxes on this date, located at the end of the photometric plateau, are indicative of a strongly reduced mass accretion rate onto the star (Fig. 10). According to magnetospheric accretion models, high velocity redshifted absorption components in the Balmer lines can still be seen for mass accretion rates as low as $10^{-9} M_{\odot} \mathrm{yr}^{-1}$ and actually become more conspicuous at lower accretion rate as the line optical depth decreases (Muzerolle et al. 2001). Weak veiling and the appearance of high velocity redshifted absorptions are therefore not necessarily contradictory.

We compared the observed $\mathrm{H} \alpha$ and $\mathrm{H} \beta$ average emission line profiles with emission line profiles computed from the magnetospheric models of Muzerolle et al. (2001). Figure 18 shows that although none of the models perfectly reproduce the observed profiles, the best fits are obtained for accretion rates in the range $10^{-8}-10^{-9} M_{\odot} \mathrm{yr}^{-1}$ and large inclination angles $\left(i \geq 60^{\circ}\right)$. The accretion rate is compatible with measured values for AA Tau, $\dot{M}=3.3 \times 10^{-9}$ and $7.1 \times 10^{-9} M_{\odot} \mathrm{yr}^{-1}$ obtained by Gullbring et al. (1998) and Valenti et al. (1993) respectively. The low inclination theoretical profiles are much more intense and narrower than the observed ones at all phases. The fact that only high inclination models fit the observed $\mathrm{H} \alpha$ and $\mathrm{H} \beta$ profiles suggests the inclination between the rotation and magnetospheric axis cannot be very large. In general, the Balmer line profiles presented a redward asymmetry (more emission in the blue than in the red wing) and large variability of the red wings, which is overall in agreement with the predictions of magnetospheric accretion models.

We noticed a time lag between the $\mathrm{H} \alpha, \mathrm{H} \beta$ and $\mathrm{He}$ I variations and the veiling: the lines formed farther away precede those formed close to the accretion shock, as expected for a perturbation propagating downwards the magnetospheric accretion column. The measured time delays are actually quite compatible with the timescale associated with free-falling gas in the magnetic funnel. Assuming purely radial motion from the disk's truncation radius $\left(r_{m} \simeq 8.8 R_{\star} \simeq 0.08 \mathrm{AU}\right.$, see $\left.\mathrm{B} 99\right)$ towards the star, the 1.08 days delay between $\mathrm{H} \alpha$ and veiling variations would correspond to gas infall from a distance of $8.5 R_{\star}$ above the stellar surface, which compares well with the size derived for AA Tau's magnetospheric cavity. The 0.44 days delay
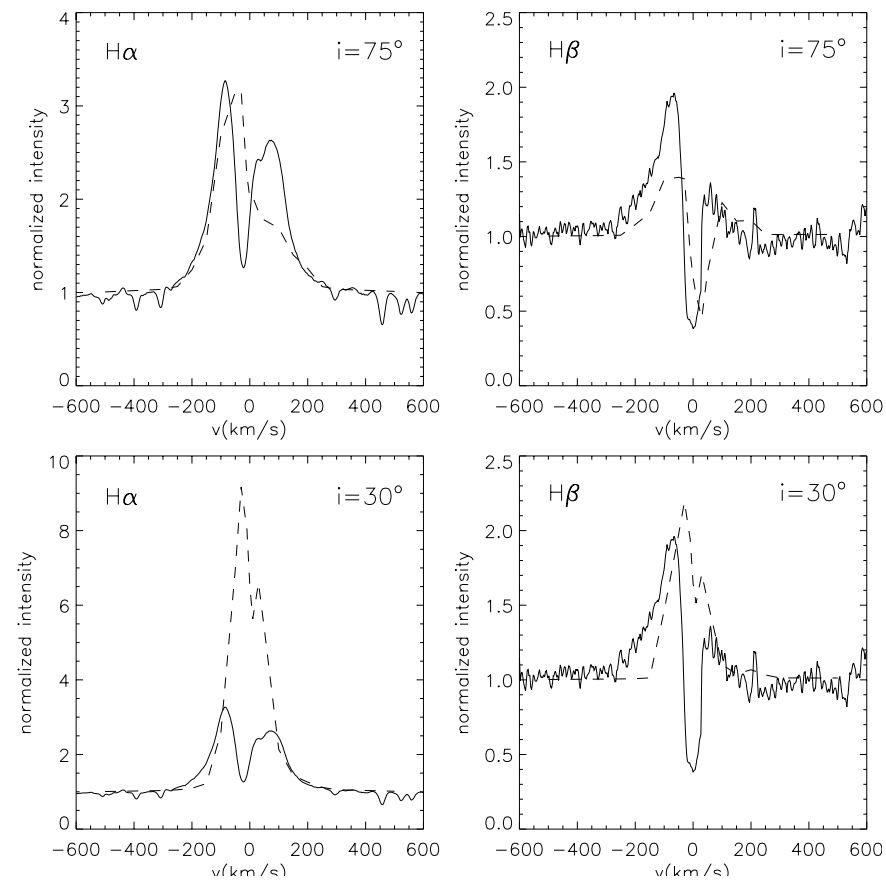

Fig. 18. Comparison between the observed $\mathrm{H} \alpha$ (left) and $\mathrm{H} \beta$ (right) average profiles (solid lines) and profiles computed from magnetospheric accretion models of Muzerolle et al. 2001 (dashed lines). The model profiles were computed for two inclinations, $75^{\circ}$ (top) and $30^{\circ}$ (bottom), a mass accretion rate of $10^{-8} M_{\odot} \mathrm{yr}^{-1}$, a maximum temperature of $7000 \mathrm{~K}$ in the accretion column and an inner and outer magnetospheric radii of 5.2 and $6 R_{\star}$, respectively, at the disk plane. Note that profiles computed at high inclination provide a much better fit than those computed at low inclination.

measured between $\mathrm{H} \beta$ and veiling would correspond to a radial scale of $\sim 6 R_{\star}$, while the much shorter 0.08 days delay measured for He I corresponds to about $1 R_{\star}$. This indicates, in agreement with the predictions of magnetospheric accretion models, that $\mathrm{H} \alpha$ is produced in the bulk of the magnetospheric cavity, while $\mathrm{H} \beta$ arises from a slightly more compact region and He I originates close to the accretion shock slightly above the stellar surface.

We also found a tight correlation between the radial velocities of the low-velocity red and blue absorption components in the $\mathrm{H} \alpha$ profile (Fig. 15). This indicates a correlation between accretion and ejection signatures, which is predicted by the magnetospheric models, since the red absorption is generally related to the accretion process and the blue absorption to outflows and winds. However, if the wind originated in a region spatially associated with the accretion funnel flow, one would expect the blueshifted and redshifted velocities to be modulated on a rotation timescale and to reach their extreme values simultaneously. In contrast, the correlation indicates that the largest blueshifted velocitites occur when the redshifted velocities are the lowest and conversely. Furthermore, we do not find evidence for a rotational modulation of the velocity of the absorption components. Instead, Fig. 10 shows a monotonic variation over two rotational cycles between JD 508 and 525. It is therefore quite unlikely that the radial velocity variations could 
result from projection effects modulated along the rotational cycle.

These variations might then reflect intrinsic changes in the velocity fields of the inflow and outflow. A tentative explanation for the observed correlation is that the location of the absorbing regions moves radially in response to simultaneous changes in the inflow and outflow optical depths. As the accretion rate and wind density both increase, the line optical depth becomes larger and the absorbing layers move upwards. This produces a lower redshifted absorption velocity since it arises in the upper, lower velocity part of the magnetic funnel flow, and a larger blueshifted velocity since it forms higher up in the accelerating wind. In this scenario, the comoving absorptions components of the $\mathrm{H}_{\alpha}$ profile would simply reflect the opposite direction of acceleration for the inflowing and outflowing material.

This interpretation, however, is not fully supported by the observations. It would require that the largest optical depth and thus accretion rate occurs around JD 524 when the lowest redshifted velocities are observed. In contrast, the line fluxes and veiling are the lowest on this date, indicative of a very weak accretion rate. Hence, we fail to find a convincing explanation of the observed correlation between the velocity of the blueshifted and redshifted $\mathrm{H} \alpha$ absorption components in the framework of a steady magnetospheric accretion model. We will come back on the origin of this correlation in the next section, when we discuss dynamical effects associated with the interaction between the inner disk and the stellar magnetic field.

\subsection{Evidence for a time-variable configuration: Beyond the idealized model}

The spectral and photometric variations of AA Tau, like those of most CTTS, are obviously more complex than would be expected from a naive axisymmetric and steady magnetospheric accretion model in which a stable stellar dipole aligned with the star's rotational axis disrupts the inner part of the accretion disk. While axial symmetry allows for the modelling of accretion-ejection structures (e.g. Shu et al. 1994; Ferreira 1997) and for the computation of line fluxes and line profiles arising in funnel flows (Muzerolle et al. 2001), the spectrophotometric monitoring of classical $\mathrm{T}$ Tauri stars reveals departures from these models.

Our previous study of the photometric variations of AA Tau already provided evidence for the accretion flow being channelled along the lines of a tilted instead of an aligned stellar dipole (B99). Since then, a surface magnetic field of order of 1-3 kG has been reported for AA Tau, with no clues however to its topology (Johns-Krull \& Valenti 2000). Evidence for a tilted dipole had previously been reported for the classical T Tauri star SU Aurigae by Johns \& Basri (1995a). The new photometric and spectroscopic data presented here are still globally consistent with the magnetospheric accretion scenario proposed for AA Tau by B99 based only on its photometric variations. However, some specific aspects of AA Tau's variability are difficult to account for by assuming a mere steady-state accretion flow along the lines of an inclined magnetosphere.
The most challenging feature in the observations reported here is the absence of a main luminosity dip around JD 521-522 (cf. Fig. 4). If these dips result from the occultation of the central star by the inner disk warp, as advocated above and in B99, how can the occulting screen disappear on a timescale of a week and reappear on the following cycle? At the time of the missing occultation, the system also exhibits little spectral variability, the veiling is minimal as are the line fluxes. All diagnostics thus suggest that the system's variability has suddenly shut off for a few days (JD 519-525, the photometric "plateau") and that the accretion flow onto the star was severely depressed at these dates.

Remarkably enough, the object was quite active on both sides of the plateau, i.e., during the previous and the following 8.2-day cycles, exhibiting both large luminosity dips and strong line profile variability. Moreover, the pattern of variability after the plateau is strinkingly similar to what it was before, as if the occulting screen had been suppressed for about the duration of a cycle and had then reformed with optical and geometrical properties quite similar to those it had before. Such a repeatability of the variability pattern would be unlikely to happen if the occultations were produced by independent, free flying dusty blobs crossing the line of sight as they orbit the star. Instead, it suggests that the properties of the absorbing material are shaped by an organized underlying structure.

In B99, we have argued that the obscuring material is to be identified with the warped inner edge of the accretion disk. The non-axisymmetric warp itself results from the response of the disk to the inclined stellar dipole (cf. Terquem \& Papaloizou 2000). The properties of the occulting screen are thus dictated by the topology of the stellar magnetic field at the disk inner edge. The stability of the warp configuration, and therefore of the observed eclipses, ultimately depends on the stability of the magnetic structure at the disk truncation radius. We thus propose that the "missing occultation" results from the perturbation and subsequent restoration of the stellar magnetic field at the disk inner edge, leading to the temporary disappearance of the disk warp and of the associated eclipse as well as to a severe reduction of the accretion flow onto the star.

The dynamical evolution of a stellar magnetosphere interacting with an accretion disk has been investigated by several recent numerical models, with applications to T Tauri stars (e.g. Romanova et al. 2002; Goodson et al. 1997; Miller \& Stone 1997). These models assume an initial dipolar configuration and predict that the stellar magnetic field lines threading the disk expand as they are twisted by differential rotation between the inner disk and the star. The inflation of stellar field lines also occurs when accreted material accumulates at the inner disk edge against the magnetosphere and builds up a pressure gradient that brings the disk truncation radius closer to the star (Romanova et al. 2002). In most models, twisted magnetic field lines eventually open, leading to an episode of strong mass outflow (e.g. Hayashi et al. 1996), and reconnect thus restoring the initial (dipolar) configuration and the associated accretion funnel flow onto the star. This evolution is found to repeat itself in a quasi-periodic manner as originally suggested by Aly \& Kuijpers (1990) with an associated timescale of order of a few 
rotation periods (e.g. Uzdensky et al. 2002a, 2002b; Goodson \& Winglee 1999; Romanova et al. 2002).

We propose that the quiescent phase of AA Tau's variability, which spans the photometric plateau and associated weak veiling and line fluxes, corresponds to a phase when the magnetosphere has expanded as described by these models, thus reducing the accretion flow onto the star as the field lines become sharply bent at the disk surface (e.g. Romanova et al. 2002). Models predict that this phase is characterized by a stronger outflow (e.g. Hayashi et al. 1996; Goodson et al. 1997), conceivably carrying away the material accumulated at the inner disk edge and thus possibly partly responsible for the disappearance of the occulting screen. The reduced accretion rate into the funnel flow may also lower the optical thickness of the absorbing material at the disk inner edge, thus producing a much shallower eclipse. Although we do not have a quantitative measure of the strength of the wind in the system, we note that the blueshifted absorption component of the $\mathrm{H} \alpha$ profile reaches below the stellar continuum during the photometric plateau while it is shallower at all other phases. This may be an indication that the outflow is indeed stronger when the accretion flow is depressed. After the inflation phase, field lines reconnect and the initial magnetic configuration is restored. A new major eclipse is thus seen around JD 528 as line fluxes start to increase again (Fig. 10). One of the differences between the major eclipses on JD 511 and JD 528 is that during the latter the system reddens while it exhibited blueing episodes during the former. This suggests that the obscuring material was partially optically thin after the restoration of the initial configuration.

This dynamical scenario of the interaction between the inner disk and the stellar magnetosphere may additionally offer an explanation to the observed correlation between the radial velocities of the blueshifted and redshifted absorption components of the $\mathrm{H} \alpha$ profile (Fig. 15). The qualitative interpretation we propose for this correlation is schematically depicted in Fig. 19. In the initial configuration, the $\mathrm{H} \alpha$ absorbing layers located in the upper part of the accretion funnel flow are nearly parallel to the line of sight, thus yielding the highest projected redshifted velocities. In the inflated phase, the field lines have strongly expanded and the same layers are now at a large angle to the line of sight, thus yielding the lowest projected redshifted velocities. In most accretion-ejection models, the outflow is nearly perpendicular to the inflow in the vicinity of the disk-magnetosphere boundary (e.g. Shu et al. 1994; Ferreira 1997). Then, the lowest projected blueshifted velocities are expected to occur in the initial configuration, when the outflow is nearly perpendicular to the line of sight, while in the inflated phase the outflow is bent towards the observer (assuming the angle between the inflow and the base of the outflow has not changed significantly) and thus yields the highest projected blueshifted velocities. Slowly evolving projection effects resulting from the inflation of the magnetosphere would thus account for the simultaneous radial velocity variations of the blue and red absorption components in the $\mathrm{H} \alpha$ profiles. This would provide a natural explanation for the fact that the highest redshifted velocities are observed when the blueshifted velocities are the lowest and conversely, an observational result

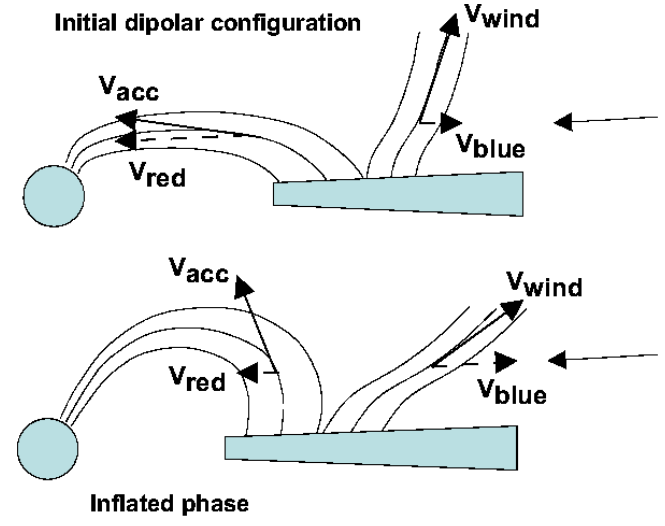

Fig. 19. A sketch of the dynamical evolution of the stellar magnetosphere interacting with the accretion disk. Top: initial dipolar configuration; bottom: the magnetosphere has expanded as magnetic field lines are twisted by differential rotation (see text). The accretion funnel flow onto the star and the nearly perpendicular outflow are schematically drawn. The line of sight to the system is highly inclined ( $i \simeq 75^{\circ}$, shown as the arrow on the right). The radial velocities of the inflow and outflow are shown. In the initial configuration, the velocity field of the absorbing layers in the accretion column is nearly parallel to the line of sight, thus yielding the highest radial velocity of the redshifted $\mathrm{H} \alpha$ absorption component. In the expanded magnetosphere configuration, the same absorbing layers make a large angle with the line of sight, thus resulting in small observed radial velocities. The outflow being nearly perpendicular to the inflow, the opposite behaviour is observed for the radial velocity of the blueshifted absorption component in the $\mathrm{H} \alpha$ line profile.

which had no straightforward explanation in the framework of a static magnetospheric accretion model.

If this interpretation is correct, the radial velocity of the redshifted Ho absorption component yields a direct measurement of the expansion of the magnetic field lines close to the diskmagnetosphere boundary, the velocity decreasing as the magnetic field line inflates. We can thus use this diagnostics to trace the evolution of the magnetospheric structure as it interacts with the disk. The radial velocity curve of the redshifted absorption component is shown in Fig. 10. As noted above, the timescale for its radial velocity variations is longer than the rotation period of the system. Figure 10 shows that the radial velocity first steadily decreases from the start of the observations on JD 506 down to its lowest value reached on JD 525, which would indicate that the magnetosphere expands over at least this time span. From JD 525 on, the radial velocity rapidly increases to reach its maximum value on JD 530 and then starts to decrease again. This phase would then correspond to the restoration of the initial magnetic configuration over JD 525-530 and the beginning of a new inflation cycle starting on JD 530.

The dynamical evolution of the magnetosphere deduced from the projected velocity of the redshifted $\mathrm{H} \alpha$ absorption component alone is globally consistent with the spectrophotometric evolution of the system: the phase of quiescent activity associated to a reduced accretion rate onto the star (photometric plateau, lowest veiling, weakest line fluxes, smallest line variability) is observed from JD 518 to 525, i.e., 
at the end of the expansion phase (JD $\leq 506-525$ ). The reapparition of the eclipses, the increase of line fluxes and of veiling all happen between JD 526 and JD 532, as the initial configuration is restored (JD 525-530). Note that three measurements strongly depart from the correlation shown in Fig. 15. They occur on JD $529.5,530.5$, and 531.5 precisely at the time the initial configuration is re-established. On JD 529.5, large variations of the radial velocity of the blueshifted component are seen to occur on a timescale of a few hours (see Fig. 10). This suggests that, superimposed onto the slowly evolving magnetospheric structure, transient ejection events occur as a new magnetospheric cycle starts.

Thus, independent observational diagnostics of the magnetospheric accretion process can be consistently accounted for by a dynamical description of the interaction between the disk and the stellar magnetosphere. This result provides strong support to the recent numerical simulations which predict a time variable behaviour of the disk-magnetosphere interface and may have important implications for the origin of the spectrophotometric variability of classical $\mathrm{T}$ Tauri stars, for the regulation of their angular momentum, and for the origin and short-term variability of outflows in young objects.

\section{Conclusions}

We have shown that the photometric and spectroscopic variations of AA Tau on days to weeks timescales are globally consistent with the concept of magnetospheric accretion in this system. Its inner accretion disk is truncated at a distance of about $0.1 \mathrm{AU}$ from the stellar surface by the strong stellar magnetosphere. The large scale magnetospheric structure is inclined relative to the disk plane which leads to the developement of a non axisymmetric warp at the inner disk edge. The warp corotates with the star and is responsible for the occurrence of the eclipses observed in AA Tau's light curve. As the eclipses proceed, high velocity inverse $\mathrm{P}$ Cygni profile are episodically observed at $\mathrm{H} \beta$ and veiling is maximum, which suggest accretion columns are seen against the hot accretion shock at this phase. The size of the magnetospheric cavity, about $8 R_{\star}$ as deduced by assuming Keplerian rotation at the inner disk edge, is consistent with the time delay we measured between lines and veiling variations as non steady accretion propagates downwards the accretion column, from the inner disk edge to the stellar surface on a timescale of one day.

The global structure of the magnetospheric accretion region appears to vary on a timescale of order of a month. We observed the sudden disappearance of eclipses together with a strong reduction of line fluxes and veiling for a few days. During this episode, the inner disk warp had apparently vanished and the accretion flow onto the star was severely depressed which suggests that the magnetic configuration at the inner disk edge has been disrupted. A few days later, the initial magnetic configuration was restored as indicated by eclipses resuming with a similar depth and shape as prior to the disruption event and line fluxes and veiling increasing again. The phase of reduced activity may correspond to a state where the field lines had strongly expanded and perhaps opened under the action of differential rotation between the inner disk and the star. In support to this interpretation, we find a smooth variation of the radial velocity of accretion and outflow diagnostics in the $\mathrm{H} \alpha$ profile which is best interpreted as reflecting the slowly inflating magnetosphere on a timescale of a month, followed by its disruption and the restoration of the initial magnetic configuration. Recent numerical simulations describe such a magnetospheric inflation process and predict they are cyclic. The results reported here may constitute the first clear evidence for the existence of magnetospheric inflation processes occurring in CTTS on a timescale of a month, though additional observations will be needed to assess their cyclic nature.

The observed variability of AA Tau is thus a complex mixture of rotational modulation by hot spots, variable circumstellar extinction, non steady accretion and varying mass loss, all of which appear to be consistent with a dynamical view of the interaction between the inner disk and an inclined large-scale stellar magnetosphere. The magnetospheric accretion process appears to be time dependent on all scales, from hours for non steady accretion to weeks for rotational modulation and months for global instabilites of the magnetospheric structure. AA Tau exhibits clear signatures of these various processes mostly because is it seen at a high inclination which maximizes the amplitude of variability. However, since AA Tau has otherwise properties of a very typical classical T Tauri star it is very likely that the processes observed in AA Tau are also instrumental in other CTTS, though more difficult to diagnose when the systems are seen at a lower inclination (e.g. Chelli et al. 1999; DeWarf et al. 2003).

The highly dynamical and time dependent nature of the magnetospheric accretion process may have implications which remain to be explored for a number of issues. For instance, the transfer of angular momentum between the star and the disk (and the wind) is certainly quite complex and time variable (Agapitou \& Papaloizou 2000). Whether this variability affects the angular momentum evolution of CTTS on the long term is however unclear, since it takes about $10^{5} \mathrm{yr}$ to the star to react to angular momentum gains or losses which will presumably smooth out the effects of short term variability. The dynamical nature of the magnetospheric accretion process may also bring clues to the origin of the short-term variability of CTTS outflows (Woitas et al. 2002; Lopez-Martin et al. 2003). It would be interesting to search for wind or jet variability on a timescale of a month, as could be expected from a cyclical evolution of the magnetospheric structure. One of the important implications of the results reported here is related to the origin of CTTS near infrared excess, which is often used as a quantitative diagnostics of mass accretion in the disk and usually modelled in the framework of standard $\alpha$-disk models. AA Tau's result suggest that the structure of the inner disk is in fact strongly modified from its interaction with the stellar magnetosphere. The development of a disk warp can be expected to considerably increase the illuminated fraction of the inner disk, possibly leading to high veiling values in the nearIR as measured by Folha \& Emerson (1999) and Johns-Krull \& Valenti (2003). Also, large scale instabilities of the magnetospheric structure at the inner disk edge can be expected to produce rapid and large near infrared variability, as observed in some systems (Carpenter et al. 2001; Eiroa et al. 2002). 
Finally, most MHD models of magnetospheric accretion developped so far assume axisymmetry and steady-state inflows/outflows. These assumptions are clearly an oversimplification of the highly dynamical processes at work in the interaction between the inner disk and the stellar magnetic field. In view of these limitations, it may not be surprising that some of the basic predictions of these models are not always confirmed by snapshot observations which catch the systems at a particular phase of their short term evolution (e.g. Johns-Krull $\&$ Gafford 2002). Whether these models remain valid to describe the evolution of these systems on the long term, once the short term variability reported here has been smoothed out over much longer timescale, remains to be seen.

Acknowledgements. We thank J. Ferreira for discussions on MHD accretion-ejection models, M. Mouchet for providing us with bibliography on possibly related processes at work in cataclysmic variables, and J. Steinacker for discussing radiative transfer issues in the framework of the magnetospheric accretion model. This work was supported by a NATO Science Program grant (PST.CLG.976194). S.H.P.A. acknowledges support from CNRS and CAPES (PRODOC program). M.F. is partially supported by the Spanish grant AYA2001-1696.

\section{References}

Agapitou, V., \& Papaloizou, J. C. B. 2000, MNRAS, 317, 273

Alencar, S. H. P., \& Basri, G. 2000, AJ, 119, 1881

Alencar, S. H. P., Johns-Krull, C. M., \& Basri, G. 2001, AJ, 122, 3335

Aly, J. J., \& Kuijpers, J. 1990, A\&A, 227, 473

Ardila, D. R., Basri, G., Walter, F. M., Valenti, J. A., \& Johns-Krull, C. M. 2002a, ApJ, 566, 1100

van Ballegooijen, A. A. 1994, Sp. Sc. Rev., 68, 299

Baranne, A., Queloz, D., Mayor, M., et al. 1996, A\&AS, 119, 373

Bardou, A., \& Heyvaerts, J. 1996, A\&A, 307, 1009

Basri, G., \& Bertout, C. 1989, ApJ, 341, 340

Bertout, C., Basri, G., \& Bouvier, J. 1988, ApJ, 330, 350

Bouvier, J., \& Bertout, C. 1989, A\&A, 211, 99

Bouvier, J., Alencar, S., \& Dougados, C. 2003, in Open Issues in Local Star Formation and Early Stellar Evolution, ed. J. Lepine, \& J. Gregorio-Hetem, in press

Bouvier, J., Chelli, A., Allain, S., et al. 1999, A\&A, 349, 619 (B99)

Camenzind, M. 1990, Rev. Mod. Astron., 3, 234

Carpenter, J. M., Hillenbrand, L. A., \& Skrutskie, M. F. 2001, AJ, 121, 3160

Chelli, A., Carrasco, L., Mújica, R., Recillas, E., \& Bouvier, J. 1999, A\&A, 345, L9

DeWarf, L. E., Sepinsky, J. F., Guinan, E. F., Ribas, I., \& Nadalin, I. 2003, ApJ, in press

Dworetsky, M. M. 1983, MNRAS, 203, 917

Edwards, S., Hartigan, P., Ghandour, L., \& Andrulis, C. 1994, AJ, 108, 1056

Eiroa, C., Oudmaijer, R. D., Davies, J. K., et al. 2002, A\&A, 384, 1038

Ferreira, J. 1997, A\&A, 319, 340

Goodson, A. P., \& Winglee, R. M. 1999, ApJ, 524, 159

Goodson, A. P., Winglee, R. M., \& Boehm, K. 1997, ApJ, 489, 199

Guenther, E. W., Lehmann, H., Emerson, J. P., \& Staude, J. 1999, A\&A, 341, 768

Gullbring, E., Hartmann, L., Briceno, C., \& Calvet, N. 1998, ApJ, 492, 323

Folha, D. F. M., \& Emerson, J. P. 1999, A\&A, 352, 517

Folha, D. F. M., \& Emerson, J. P. 2001, AA, 365, 90
Hartigan, P., Edwards, S., \& Ghandour, L. 1995, ApJ, 452, 736

Hartmann, L., Hewett, R., \& Calvet, N. 1994, ApJ, 426, 669

Hartmann, L., Hewett, R., Stahler, S., \& Mathieu, R. D. 1986, ApJ, 309,275

Hartmann, L., \& Stauffer, J. R. 1989, AJ, 97, 873

Hayashi, M. R., Shibata, K., \& Matsumoto, R. 1996, ApJ, 468, L37

Horne, J. H., \& Baliunas, S. L. 1986, ApJ, 302, 757

Ilyin, I. V. 2000, High resolution SOFIN CCD echelle spectroscopy, Ph.D. Thesis, University of Oulu

Johns, C. M., \& Basri, G. 1995a, ApJ, 449, 341

Johns, C. M., \& Basri, G. 1995b, AJ, 109, 2800

Johns-Krull, C. M., \& Gafford, A. D. 2002, ApJ, 573, 685

Johns-Krull, C. M., \& Valenti, J. A. 2003, ApJ, in press

Johns-Krull, C. M., \& Valenti, J. A. 2000, Stellar Clusters and Associations: Convection, Rotation, and Dynamos, ASP Conf. Ser., 198, 371

Johns-Krull, C. M., Valenti, J. A., \& Koresko, C. 1999, ApJ, 516, 900

Johns-Krull, C. M., Valenti, J. A., Saar, S. H., \& Hatzes, A. P. 2001, ASP Conf. Ser., 223, 11th Cambridge Workshop on Cool Stars, Stellar Systems and the Sun, 11, 521

Kaufer, A., \& Pasquini, L. 1998, SPIE, 3355, 844

Kaufer, A., Stahl, O., Tubbesing, S., et al. 2000, SPIE, 4008, 459

Kenyon, S. J., \& Hartmann, L. 1995, ApJs, 101, 117

Koenigl, A. 1991, ApJ, 370, L39

Lai, D. 1999, ApJ, 524, 1030

Lin, D. N. C., Bodenheimer, P., \& Richardson, D. C. 1996, Nature, 380, 606

Lopez-Martin, L., Cabrit, S., \& Dougados, C. 2003, A\&A, 405, L1

Lynden-Bell, D., \& Boily, C. 1994, MNRAS, 267, 146

Ménard, F., \& Bertout, C. 1999, NATO ASIC Proc. 540, The Origin of Stars and Planetary Systems, 341

Ménard, F., Bouvier, J., Dougados, C., Melnikov, S. Y., \& Grankin, K. 2003, A\&A, 409, 163

Meyer, M. R., Calvet, N., \& Hillenbrand, L. A. 1997, AJ, 114, 288

Miller, K. A., \& Stone, J. M. 1997, ApJ, 489, 890

Montmerle, T., Grosso, N., Tsuboi, Y., \& Koyama, K. 2000, ApJ, 532, 1097

Movsessian, T., Boulesteix, J., Gach, J.-L., \& Zaratsian, S. 2000, Baltic Astronomy, 9, 652

Muzerolle, J., Hartmann, L., \& Calvet, N. 1998, AJ, 116, 455

Muzerolle, J., Hartmann, L., \& Calvet, N. 2001, ApJ, 550, 944

Muzerolle, J., Calvet, N., \& Hartmann, L. 2001, ApJ, 550, 944

Oliveira, J. M., Foing, B. H., van Loon, J. T., \& Unruh, Y. C. 2000, A\&A, 362, 615

Petrov, P. P., Shcherbakov, V. A., Berdyugina, et al. 1994, A\&AS, 107, 9

Petrov, P. P., Gullbring, E., Ilyin, I., et al. 1996, A\&A, 314, 821

Petrov, P. P., Gahm, G. F., Gameiro, J. F., et al. 2001, A\&A, 369, 993

Queloz, D. 1995, Ph.D. Thesis, Université de Genève

Queloz, D., Allain, S., Mermilliod, J.-C., Bouvier, J., \& Mayor, M. 1998, A\&A, 335, 183

Romanova, M. M., Ustyugova, G. V., Koldoba, A. V., \& Lovelace, R. V. E. 2002, ApJ, 578, 420

Savage, B. D., \& Mathis, J. S. 1979, ARA\&A, 17, 73

Scargle, J. D. 1982, ApJ, 263, 835

Shevchenko, V. S., Grankin, K. N., Ibragimov, M. A., et al. 1991, Informational Bulletin on Variable Stars, 3652, 1

Shu, F., Najita, J., Ostriker, E., et al. 1994, ApJ, 429, 781

Smith, K., Pestalozzi, M., Güdel, M., Conway, J., \& Benz, A. O. 2003 , A\&A, 406, 957

Stout-Batalha, N. M., Batalha, C. C., \& Basri, G. 2000, ApJ, 532, 474

Terquem, C., \& Papaloizou, J. C. B. 2000, A\&A, 360, 1031 
Tuominen, I., Ilyin, I., \& Petrov, P. 1999, in Astrophysics with the NOT, Proc. of the Conference held in Turku in August 12-15, 1998, ed. H. Karttunen, \& V. Piirola, University of Turku, 47 Uzdensky, D. A., Königl, A., \& Litwin, C. 2002a, ApJ, 565, 1191 Uzdensky, D. A., Königl, A., \& Litwin, C. 2002b, ApJ, 565, 1205 Valenti, J. A. 1994, Ph.D. Thesis, Univ. of California, Berkeley Valenti, J. A., Basri, G., \& Johns, C. M. 1993, AJ, 106, 2024
Vogt, S. S. 1987, PASP, 99, 1214

Vrba, F. J., Chugainov, P. F., Weaver, W. B., \& Stauffer, J. S. 1993, AJ, 106,1608

Vrba, F. J., Rydgren, A. E., Chugainov, P. F., Shakovskaia, N. I., \& Weaver, W. B. 1989, AJ, 97, 483

Woitas, J., Ray, T. P., Bacciotti, F., Davis, C. J., \& Eislöffel, J. 2002, ApJ, 580, 336 